\title{
Aging reduces calreticulin expression and alters spontaneous calcium signals in astrocytic endfeet of the mouse dorsolateral striatum
}

Abbreviated Title: Aging alters astrocyte endfoot $\mathrm{Ca}^{2+}$ signals

Authors: Sara M. Zarate ${ }^{1}$, Taylor E. Huntington ${ }^{1,3}$, Pooneh Bagher ${ }^{2}$ and Rahul Srinivasan ${ }^{1,3}$

Author affiliations: Department of Neuroscience \& Experimental Therapeutics ${ }^{1}$ and

Department of Medical Physiology², Texas A\&M University College of Medicine, 8447

Riverside Pkwy, Bryan, TX 77807, USA. Texas A\&M Institute for Neuroscience (TAMIN),

Texas A\&M University, College Station, TX 77843, USA ${ }^{3}$.

Corresponding Author: Rahul Srinivasan, Department of Neuroscience \& Experimental Therapeutics, Texas A\&M University College of Medicine, Bryan, TX 77807-3260, USA

Email: $\underline{\text { rahul@tamu.edu }}$

Acknowledgements: This work was partially funded by R01HL155618 to PB and an American Diabetes Association Junior Faculty Development Award, 1-19-JDF-111 to PB and by a National Institutes of Health (NIH) research grant, R01NS115809 to RS. 


\title{
Double-spaced pages (Introduction + Materials and Methods + \\ Results + Discussion + References + Figure Legends + Figures $)=36$
}

\section{Manuscript word count}

Introduction + Materials and Methods + Results + Discussion: 4335

\author{
Abstract: 170 \\ Introduction: 616 \\ Materials and Methods: 1215
}

Results: 1553

Discussion: 951

Figure legends: 1590

Number of Figures: 10

Number of Tables: 0

Contents of Supplemental Material: 8 supplemental movies

Number of References: 50

Conflict of interest statement: Authors declare no competing financial interests. 


\section{Author contributions: SMZ performed all experiments and analyses, created figures,} and contributed to writing and editing the manuscript. TEH created the tools and techniques to measure $\mathrm{Ca}^{2+}$ influx into mitochondria, and contributed to editing the manuscript. PB designed and supervised experiments and associated analysis and contributed to editing the manuscript. RS conceptualized, designed and coordinated the study, trained and supervised SMZ and TEH, designed and supervised experiments, advised on figures, provided resources and funding, and wrote the manuscript with SMZ

\section{CRediT author statement}

Sara M. Zarate: Methodology, Validation, Formal analysis, Investigation, Writing - Original draft, Writing - Review \& Editing, Visualization

Taylor E. Huntington: Methodology, Writing - Review \& Editing

Pooneh Bagher: Conceptualization, Methodology, Formal Analysis, Writing - Review \& Editing

Rahul Srinivasan: Conceptualization, Methodology, Validation, Formal Analysis, Investigation, Resources, Writing - Original draft, Writing - Review \& Editing, Visualization, Supervision, Project administration, Fund ing acquisition 


\section{Abstract}

Aging-related impairment of the blood brain barrier (BBB) and neurovascular unit (NVU) increases risk for neurodegeneration. Among the various cells participating in BBB and NVU function, spontaneous $\mathrm{Ca}^{2+}$ signals in astrocytic endfeet are crucial for maintaining $\mathrm{BBB}$ and NVU integrity. To assess if aging is associated with changes in spontaneous $\mathrm{Ca}^{2+}$ signals within astrocytic endfeet of the dorsolateral striatum (DLS), we expressed a genetically encoded $\mathrm{Ca}^{2+}$ indicator, Lck-GCaMP6f in DLS astrocytes of young (3-4 month) and aging (20-24 month) mice. Compared to young mice, endfeet in the DLS of aging mice demonstrated a decrease in calreticulin (CALR) expression, and dramatic alterations in the dynamics of endfoot membraneassociated and mitochondrial $\mathrm{Ca}^{2+}$ signals. While young mice required both extracellular and endoplasmic reticulum (ER) $\mathrm{Ca}^{2+}$ sources for generating endfoot $\mathrm{Ca}^{2+}$ signals, aging mice showed exclusive dependence on $\mathrm{ER} \mathrm{Ca}^{2+}$. These data suggest that aging is associated with significant changes in $\mathrm{Ca}^{2+}$ buffers and $\mathrm{Ca}^{2+}$ signals within astrocytic endfeet, which has important implications for understanding mechanisms involved in aging-related impairment of the BBB and NVU.

\section{Keywords:}

Astrocytes, Endfeet, Calcium, Endoplasmic reticulum, Calreticulin, Parkinson's disease

\section{Abbreviations:}

BBB - blood brain barrier, NVU - neurovascular unit, BV - blood vessel, CALR - calreticulin, ER - endoplasmic reticulum, AAV - adeno-associated virus, PD - Parkinson's disease, AD Alzheimer's disease, aCSF - artificial cerebrospinal fluid, DLS - dorsolateral striatum, AQP4 aquaporin 4 


\section{Graphical abstract}

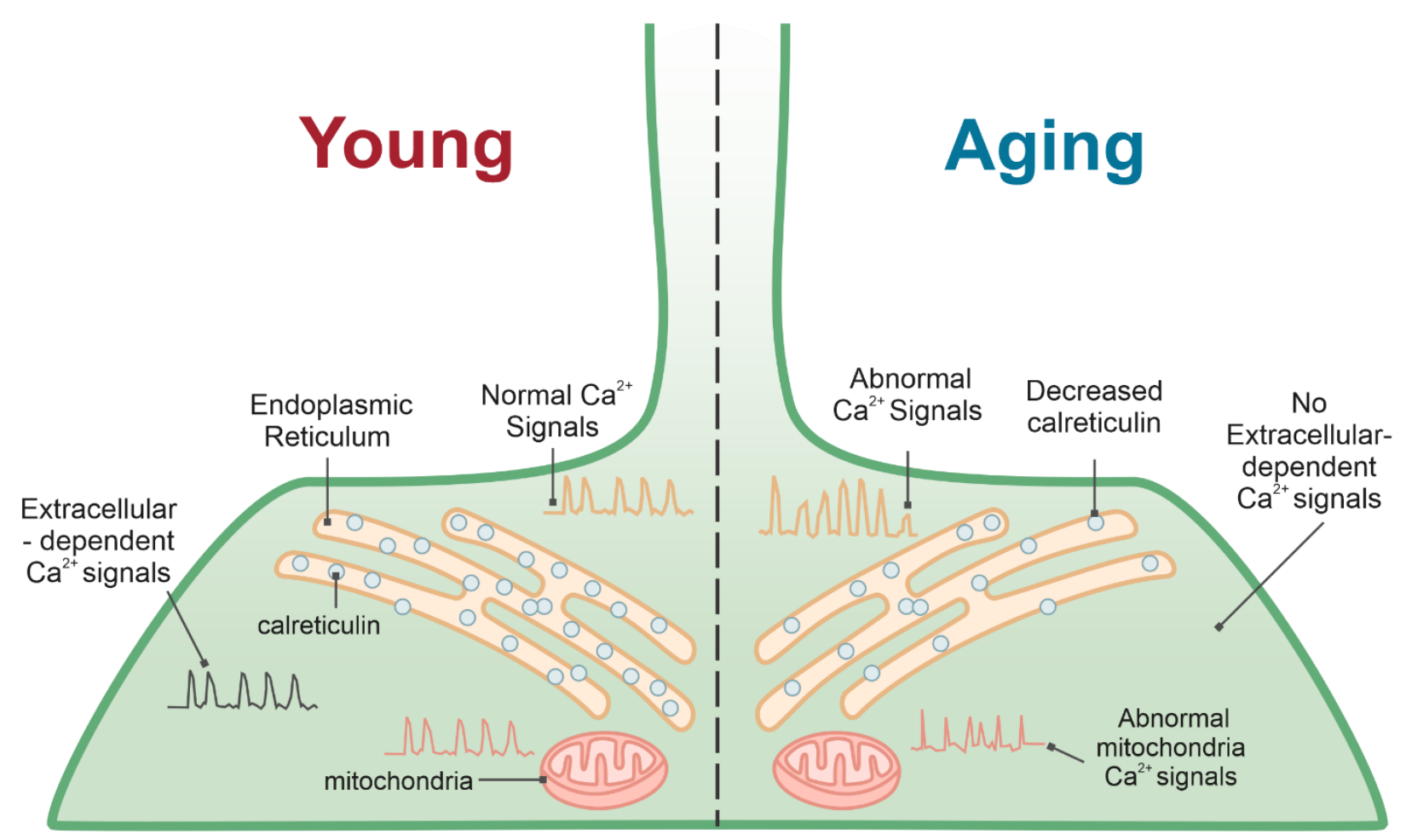

\section{Highlights}

- Aging mice show reduced calreticulin expression in astrocytic endfeet

- Aging astrocytic endfeet show dramatic changes in spontaneous $\mathrm{Ca}^{2+}$ activity

- $\mathrm{Ca}^{2+}$ signals in aging endfeet depend exclusively on endoplasmic reticulum $\mathrm{Ca}^{2+}$ 


\section{Introduction}

Advanced age is the single greatest risk factor for neurodegenerative conditions such as Parkinson's disease (PD), Alzheimer's disease (AD) (Hou et al., 2019), stroke (Leritz et al., 2011; Yousufuddin and Young, 2019), and dementia (Irwin et al., 2018). A large body of evidence strongly suggests that aging-related impairment of the blood brain barrier (BBB) and neurovascular unit (NVU) potentiates, and possibly triggers neurodegenerative processes in the brain (Cabezas et al., 2014; Cai et al., 2017; Govindpani et al., 2019; Grammas et al., 1999; Ott et al., 2018; Sweeney et al., 2019; Yu et al., 2020; Zhou et al., 2020). Among the multiple brain regions prone to neurodegeneration, the striatum is particularly vulnerable to neurovascular dysfunction and neurodegeneration because of its rich vasculature supply (Feekes and Cassell, 2006). This notion is supported by reports showing a higher percentage of lacunar infarcts in the striatum compared to other brain regions (Feekes et al., 2005), increased propensity for accumulating toxic striatal protein aggregates (Duda et al., 2002; Hanseeuw et al., 2019), and an increase in striatal BBB permeability during PD, AD and stroke (Gray and Woulfe, 2015; Haley and Lawrence, 2017; Sweeney et al., 2018). Furthermore, aging-related changes in the striatal BBB and NVU can lead to parkinsonian symptoms (Rosso et al., 2018), enlarged perivascular spaces (Chung et al., 2021), and PD due to dopaminergic neuron loss (Ivanidze et al., 2020). Although these studies indicate that striatal BBB and NVU dysfunction are important contributing factors for various forms of neurodegeneration, we know very little about the specific molecular mechanisms involved in altering BBB and NVU function during aging.

The BBB and NVU are comprised of distinct cellular elements, viz., end othelial cells, pericytes and astrocytes, each of which plays important and specific roles in neurovascular function (Garcia and Longden, 2020; Mishra et al., 2016; Pandit et al., 2020; Villabona-Rueda et 
al., 2019). Among these cell types, astrocytes are unique in that they simultaneously contact neurons and the vasculature, thus enabling these cells to regulate blood flow in response to brain activity (Attwell et al., 2010; Belanger et al., 2011; Heithoff et al., 2021; Howarth, 2014). Astrocytes perform this vital function via spontaneous $\mathrm{Ca}^{2+}$ signals within endfeet that completely ensheath capillaries (Girouard et al., 2010; Zhang et al., 2019). Indeed, $\mathrm{Ca}^{2+}$ signals in astrocytic endfeet have been shown to mediate vascular repair after injury (Gobel et al., 2020), regulate brain volume (Eilert-Olsen et al., 2019), as well as maintain NVU coupling (Dunn et al., 2013). Thus, at a functional level, astrocytic endfoot $\mathrm{Ca}^{2+}$ signals govern critical aspects of BBB integrity and NVU function. It follows that aging-related changes in striatal astrocytic end foot $\mathrm{Ca}^{2+}$ signals likely contribute to BBB and NVU dysfunction during neurodegeneration. Based on the strong body of evidence pointing to striatal vascular dysfunction in various forms of agingrelated neurodegeneration as well as a central role for astrocytic endfoot $\mathrm{Ca}^{2+}$ signals in maintaining BBB integrity and NVU function, we asked if aging is associated with alterations in spontaneous astrocytic endfoot $\mathrm{Ca}^{2+}$ events within the dorsolateral striatum (DLS) of acutely extracted live brain slices from young and aging mice.

We show that when compared to young mice, DLS brain slices of aging mice display dramatic alterations in the kinetics, dynamics, and sources of spontaneous membrane-associated $\mathrm{Ca}^{2+}$ events, as well as changes in $\mathrm{Ca}^{2+}$ influx into astrocytic endfoot mitochondria. These agingrelated alterations in endfoot $\mathrm{Ca}^{2+}$ events are associated with significant reductions in the expression of a major endoplasmic reticulum (ER) $\mathrm{Ca}^{2+}$ buffering protein, calreticulin (CALR). Our findings have important implications for understanding how an aging-related reduction in CALR expression can alter astrocytic endfoot $\mathrm{Ca}^{2+}$ signals in the $\mathrm{BBB}$ and NVU, eventually resulting in vascular dysfunction during aging and aging-related neurodegeneration. 


\section{Materials and Methods}

\section{Mice}

Male and female C57BL/6J mice (Jackson laboratory, Bar Harbor, ME, USA) were used for all experiments. Young mice were 3-4 months of age, and aging mice were 20-24 months of age. Mice were aged in house, in the animal vivarium at Texas A\&M University. All experiments were performed in accordance with Texas A\&M University IACUC regulations and protocols. Food and water were provided ad libitum. All mice were maintained on a $12 \mathrm{~h}$ lightdark cycle.

\section{Adeno-associated virus (AAV) injection into the DLS of mice}

Young and aging mice were deeply anesthetized using isoflurane dispensed from a SomnoSuite Low Flow Anesthesia System (Kent Scientific, Torrington, CT), and a craniotomy was performed as previously described (Huntington and Srinivasan, 2021; Jiang et al., 2014). AAVs were injected into the right DLS using a custom-made glass injection pipette with a $6 \mu \mathrm{m}$ tip diameter, at a rate of $750 \mathrm{nl} / \mathrm{min}$ using a Harvard Apparatus Pump 11 Pico Plus Elite, 7041506 (Harvard Apparatus, Holliston, MA). To image membrane-associated astrocyte endfoot $\mathrm{Ca}^{2+}$ events in young and aging mice, $1 \mu \mathrm{l}$ of AAV2/5-GfaABC1D-Lck-GCaMP6f $\left(10^{13}\right.$ genome copies/ml) (Addgene viral prep \# 52924-AAV5) was injected into the DLS. The Lck motif at the N-terminal end of GCaMP6f enables plasma-membrane insertion of GCaMP, and visualization of membrane-associate $\mathrm{Ca}^{2+}$ signals (Srinivasan et al., 2016). To image astrocyte endfoot mitochondrial $\mathrm{Ca}^{2+}$ events in young and aging mice, $2 \mu \mathrm{l}$ of AAV2/5-GfaABC1D-mito7GCaMP6f (10 13 genome copies/ml) (Huntington and Srinivasan, 2021) was injected into the 
DLS. Coordinates for stereotaxic injection of AAVs were $0.8 \mathrm{~mm}$ rostral to the bregma, $2 \mathrm{~mm}$ lateral to the midline and $2.4 \mathrm{~mm}$ ventral to the pial surface.

\section{Confocal imaging of acute brain slices}

Young and aging mice were deeply anesthetized with isoflurane and the left ventricle of the heart was rapidly accessed by opening the chest cavity. $200 \mu \mathrm{l}$ of DyLight 594 labeled Lycopersicon esculentum lectin (tomato lectin, TL) (DL-1177, Vector Laboratories, Burlingame, CA) was injected into the apex of the beating left ventricle. $2-3 \mathrm{~min}$ following TL injection into the left ventricle, the mouse was decapitated and the brain was extracted, then blocked within 2 min. for obtaining live brain slices. $400 \mu \mathrm{m}$ coronal striatal slices were cut using a Microslicer $01 \mathrm{~N}$ (Ted Pella) in a solution comprising $194 \mathrm{mM}$ sucrose, $30 \mathrm{mM} \mathrm{NaCl}, 4.5 \mathrm{mM} \mathrm{KCl,} 1.2 \mathrm{mM}$ $\mathrm{NaH}_{2} \mathrm{PO}_{4}, 26 \mathrm{mM} \mathrm{NaHCO} 3,10 \mathrm{mM}$ D-glucose, and $1 \mathrm{mM} \mathrm{MgCl}_{2}$ bubbled with $95 \% \mathrm{O}_{2}$ and $5 \%$ $\mathrm{CO}_{2}$. Live brain slices were incubated in artificial cerebrospinal fluid (aCSF) composed of 126 $\mathrm{mM} \mathrm{NaCl}, 2.5 \mathrm{mM} \mathrm{KCl}, 1.24 \mathrm{mM} \mathrm{NaH}_{2} \mathrm{PO}_{4}, 1.3 \mathrm{mM} \mathrm{MgCl}_{2}, 2.4 \mathrm{mM} \mathrm{CaCl} 2,26 \mathrm{mM} \mathrm{NaHCO}$, $10 \mathrm{mM}$ D-glucose at $33^{\circ} \mathrm{C}$ for $30 \mathrm{~min}$ and then maintained at $23^{\circ} \mathrm{C}$ in aCSF for the duration of the experiment.

Live mouse striatal brain slices were imaged with an Olympus FV1200 upright laserscanning confocal microscope using a 40x water immersion objective lens (NA 0.8), and a digital zoom of 3x. We used a $488 \mathrm{~nm}$ excitation wavelength at $10 \%$ of maximum intensity of a $100 \mathrm{~mW}$ Argon laser to record membrane-associated astrocyte endfoot and mitochondrial $\mathrm{Ca}^{2+}$ events. A $25 \mathrm{~mW}$ HeNe $594 \mathrm{~nm}$ excitation laser line at $10 \%$ of maximum intensity was used to visualize blood vessels (BVs) and localize astrocytic endfoot $\mathrm{Ca}^{2+}$ events in the DLS. All BVs reported in this study were capillaries with a diameter range of 5 to $10 \mu \mathrm{m}$. Endfoot membrane- 
associated and mitochondrial $\mathrm{Ca}^{2+}$ events in close proximity to TL-labeled BVs were identified as astrocytic endfoot $\mathrm{Ca}^{2+}$ events and imaged for $10 \mathrm{~min}$ at 1 frame per second (FPS). Imaging parameters (laser intensity, HV, gain, offset, aperture diameter) were maintained constant across all imaging sessions.

Imaging of $\mathrm{Ca}^{2+}$ events in astrocytic endfeet was performed by first imaging spontaneous $\mathrm{Ca}^{2+}$ events near BVs, followed by sequential depletion of extracellular $\mathrm{Ca}^{2+}$ and $\mathrm{ER} \mathrm{Ca}^{2+}$ in the slice. To deplete extracellular $\mathrm{Ca}^{2+}$, spontaneous $\mathrm{Ca}^{2+}$ events were recorded for 5 min in bath perfused aCSF, followed by bath perfusion of zero $\mathrm{Ca}^{2+} \mathrm{aCSF}$, in which $\mathrm{CaCl}_{2}$ was omitted. To deplete ER $\mathrm{Ca}^{2+}$ stores, $20 \mu \mathrm{M}$ cyclopiazonic acid (CPA, Abcam, Cambridge, MA, ab120300) was perfused into the bath for $15 \mathrm{~min}$, after which $\mathrm{Ca}^{2+}$ events were recorded for $5 \mathrm{~min}$ from the same field of view.

\section{Immunohistochemistry}

Mice were deeply anesthetized with isoflurane and transcardially perfused with 1X PBS, followed by $10 \%$ formalin. Brains were extracted and stored in $10 \%$ formalin for $48 \mathrm{~h}$ at $4{ }^{\circ} \mathrm{C}$ then dehydrated in 30\% sucrose in 1X PBS (Sigma, St Louis, MO, cat\# S7903) for 48 h. A Microm HM 550 cryostat was used to cut $40 \mu \mathrm{m}$ coronal sections of the striatum that were stored at $4{ }^{\circ} \mathrm{C}$ in $0.01 \%$ sodium azide (Sigma, cat\# S2002) until the day of immunostaining. Sections were washed $3 x$ in $1 X$ PBS, then permeabilized and blocked in $0.5 \%$ Triton $X-100$ and $10 \%$ normal goat serum at room temperature for $1 \mathrm{~h}$. To prevent cross reactivity, sections were sequentially stained with CALR and AQP4 primary and second ary antibodies. Sections were first incubated overnight at $4^{\circ} \mathrm{C}$ in rabbit anti-CALR (1:500, ThermoFisher, Waltham, MA, cat\# PA3900) then goat anti-rabbit Alexa Fluor 488 (1:2000, Abcam, cat\# ab150077) secondary antibody for $2 \mathrm{~h}$ at room temperature. After 3 washes in $1 \mathrm{X}$ PBS, sections were stained with 
rabbit anti-AQP4 (1:200, Alomone, cat\# AQP-004) overnight at $4^{\circ} \mathrm{C}$, followed by goat antirabbit Alexa Fluor 594 (1:2000, Abcam, cat\# ab150176) for 2 h at room temperature. Following staining, sections were mounted on glass slides and imaged using an FV1200 inverted confocal microscope equipped with a 60x oil immersion objective.

\section{Analysis of $\mathrm{Ca}^{2+}$ events in astrocytic endfeet}

Membrane-associated and mitochondrial astrocytic endfoot $\mathrm{Ca}^{2+}$ events were identified as $\mathrm{Ca}^{2+}$ events occurring immediately adjacent to the abluminal side of TL-labeled DLS BVs. Regions of interest (ROIs) for endfoot $\mathrm{Ca}^{2+}$ events identified in this way were generated as previously described, using the ImageJ plugin, GECIquant (Srinivasan et al., 2015). dF/F traces of GCaMP6f fluorescent signals were generated from the acquired ROIs and analyzed with Minianlysis 6.0.07 (Synaptosoft) to generate frequency (events/min), amplitude (dF/F), and halfwidth (s) values for each trace.

For expanding endfoot $\mathrm{Ca}^{2+}$ waves, the velocity $(\mu \mathrm{m} / \mathrm{s})$, distance traveled $(\mu \mathrm{m})$, and area $\left(\mu \mathrm{m}^{2}\right)$ of membrane-associated astrocytic end foot $\mathrm{Ca}^{2+}$ events were analyzed using ImageJ. All confocal movies for analysis of these parameters were acquired at a frame rate of 1 FPS. For analyzing velocity, the distance covered by endfoot $\mathrm{Ca}^{2+}$ events was determined by using the line tool in ImageJ to trace the length of $\mathrm{Ca}^{2+}$ events along the corresponding $\mathrm{BV}$ across multiple frames, then divided by time. Distance traveled corresponds to the maximum length attained by any given $\mathrm{Ca}^{2+}$ event along a $\mathrm{BV}$. The area of propagating $\mathrm{Ca}^{2+}$ events was quantified using the polygon tool in ImageJ. Using the polygon tool, the maximum area attained by a propagating $\mathrm{Ca}^{2+}$ event along the BV was manually outlined, and the area was measured. 


\section{Sampling and statistics}

Individual groups consist of all the DLS endfoot ROIs from 3 to 7 mice. Statistics were performed using Origin 2019 (OriginLab, Northampton, MA). For statistical testing, datasets were first tested for normality. Non-normal datasets were subjected to either a Mann-Whitney or paired sample Wilcoxon signed rank test. A two-sample t test was used to compare normally distributed datasets. $p<0.05$ was considered statistically significant. Sample sizes, statistical tests used, and exact p-values are reported in figures and figure legends.

\section{Results}

\section{Aging alters multiple parameters of spontaneous membrane-associated astrocytic endfoot $\mathrm{Ca}^{2+}$ events in the DLS}

To measure spontaneous membrane-associated astrocytic endfoot $\mathrm{Ca}^{2+}$ events, the DLS of either young or aging mice was injected with an AAV expressing membrane localized GCaMP6f (Lck-GCaMP6f), driven by an astrocyte-specific GfaABC1D promoter (AAV2/5GfaABC1D-Lck-GCaMP6f). Intraventricular administration of red fluorescent TL just prior to live brain slicing enabled visualization of BVs in the DLS (Figure 1A), while Lck-GCaMP6f was used to visualize astrocytic endfoot $\mathrm{Ca}^{2+}$ events (Figure 1B). Confocal imaging of live striatal brain slices co-labeled with TL in BVs and Lck-GCaMP6f in astrocytic endfeet revealed that both young and aging mice exhibited robust spontaneous endfoot $\mathrm{Ca}^{2+}$ events in ROIs immediately adjacent to TL labeled BVs in the DLS (Figures 1B and 1C; supplementary movies 1 and 2).

We found that the number of endfoot $\mathrm{Ca}^{2+} \mathrm{ROIs}$ was greater in young mice when compared to aging mice $(3.2 \pm 0.53 \mathrm{ROIs}$ per $\mathrm{BV}$ in young and $2.15 \pm 0.28 \mathrm{ROIs}$ per $\mathrm{BV}$ in 
aging mice), while the average area of endfoot $\mathrm{Ca}^{2+} \mathrm{ROI}$ in aging mice was $\sim 36 \%$ larger than in young mice $\left(\mathrm{ROI}\right.$ area $=32.79 \pm 4.3 \mu \mathrm{m}^{2}$ in young and $50.6 \pm 5.15 \mu \mathrm{m}^{2}$ in aging mice) (Figure 1D). Next, we measured the kinetics (frequency, amplitude, and half width) of DLS astrocytic endfoot $\mathrm{Ca}^{2+}$ events in young and aging mice. Young mice demonstrated an average endfoot $\mathrm{Ca}^{2+}$ event frequency of 1 event/min, which was $20 \%$ faster than aging mice (Figure 2A). Cumulative frequency distributions of endfoot $\mathrm{Ca}^{2+}$ events in young mice revealed that $90 \%$ of events occurred between 0.2 and 1.6 events/min that segregated into 8 regularly spaced intervals. By contrast, in aging mice, $80 \%$ of all $\mathrm{Ca}^{2+}$ events occurred between 0.2 and 1.2 events/min, and segregated into more than 20 smaller spaced intervals (Figure $2 \mathrm{~B}$ ). Endfoot $\mathrm{Ca}^{2+}$ events in young mice displayed a constant frequency interval of 0.2 events/min, while endfoot $\mathrm{Ca}^{2+}$ events in aging mice showed a significantly shorter interval of $\sim 0.1$ events/min, which was highly variable (Figure 2C). Aging mice also showed a $67 \%$ greater increase in $\mathrm{Ca}^{2+}$ event amplitude and a $40 \%$ greater increase in $\mathrm{Ca}^{2+}$ event half width when compared to young mice (Figure 2D).

Endfoot $\mathrm{Ca}^{2+}$ events in young and aging mice occurred as expanding waves traveling along the length of BVs (Figure 3A; supplementary movies 1 and 2). For these expanding $\mathrm{Ca}^{2+}$ waves, the velocity, distance, and area covered were measured. Compared to young mice, aging mice showed a significant increase in the expansion velocity of end foot $\mathrm{Ca}^{2+}$ events $(10.98 \pm$ $1.45 \mu \mathrm{m} / \mathrm{s}$ in aging mice and $5.77 \pm 0.93 \mu \mathrm{m} / \mathrm{s}$ in young mice) but showed no differences either in the distance covered by $\mathrm{Ca}^{2+}$ waves or the total area of $\mathrm{Ca}^{2+}$ waves (Figure $3 \mathrm{~B}$ ).

Taken together, these data show that aging significantly alters multiple aspects of the kinetics of spontaneous endfoot $\mathrm{Ca}^{2+}$ events in the DLS. 


\section{Aging increases the dependence of astrocytic endfoot signals on ER $\mathrm{Ca}^{2+}$ stores}

Having found that the DLS of aging mice show significant alterations in spontaneous astrocytic endfoot $\mathrm{Ca}^{2+}$ signals, we sought to determine if there were also differences in the extent to which endfoot $\mathrm{Ca}^{2+}$ events in young versus aging mice depend on extracellular and intracellular $\mathrm{Ca}^{2+}$ stores.

Extracellular and intracellular $\mathrm{Ca}^{2+}$ stores were sequentially depleted and astrocytic endfoot $\mathrm{Ca}^{2+}$ events were recorded. In young mice, depletion of extracellular $\mathrm{Ca}^{2+}$ with zero $\mathrm{Ca}^{2+}$ aCSF caused a significant $23 \%$ decrease in $\mathrm{Ca}^{2+}$ event frequency (Figure 4A and B;

supplemental movie 1) with no effect on the amplitude and half width of $\mathrm{Ca}^{2+}$ events (Figure $5 \mathrm{~A})$. Depletion of ER $\mathrm{Ca}^{2+}$ stores in young mouse brain slices with $20 \mu \mathrm{M}$ of the $\mathrm{Ca}^{2+}$-ATPase SERCA pump inhibitor, CPA, for 15 minutes dramatically reduced $\mathrm{Ca}^{2+}$ event frequency by $\sim 40 \%$ (Figure 4A and B). In addition, young mice showed a 78\% decrease in the amplitude and a $43 \%$ decrease in the half width of $\mathrm{Ca}^{2+}$ events that persisted after CPA exposure (Figure 5C).

For aging mice, zero $\mathrm{Ca}^{2+} \mathrm{aCSF}$ did not affect $\mathrm{Ca}^{2+}$ event frequency (Figure 4D and E; supplemental movie 2) or amplitude but caused a small decrease in half width (Figure 5B). However, following CPA treatment, DLS brain slices of aging mice showed a dramatic $75 \%$ reduction in $\mathrm{Ca}^{2+}$ event frequency (Figure 4D and E). Aging mice also demonstrated a 71\% decrease in amplitude and a $30 \%$ decrease in half width of the $\mathrm{Ca}^{2+}$ events that persisted after CPA application (Figure 5D).

These data show that while young mice rely on both extracellular and intracellular $\mathrm{Ca}^{2+}$ stores, aging mice almost exclusively rely on $\mathrm{ER} \mathrm{Ca}^{2+}$ for generating spontaneous endfoot $\mathrm{Ca}^{2+}$ events in the DLS (Figure 4C and F). 


\section{Astrocytic endfeet in the DLS of aging mice demonstrate significant reductions in CALR}

Based on the finding that aging mice exclusively rely on $\mathrm{ER} \mathrm{Ca}^{2+}$ for generating endfoot $\mathrm{Ca}^{2+}$ events (Figures 4 and 5), we rationalized that aging may be associated with an alteration in $\mathrm{ER} \mathrm{Ca}^{2+}$ buffering. To test this hypothesis, young and aging striatal brain sections were coimmunostained for the major ER-localized $\mathrm{Ca}^{2+}$ buffering protein, CALR (Nakamura et al., 2001), and the astrocytic endfoot marker, AQP4. In both young and aging mice, AQP4 labeling clearly outlined BVs, while CALR staining appeared as punctate structures throughout AQP4 stained endfeet as well as the surrounding neuropil (Figure 6A). CALR positive punctae that colocalized with AQP4 were used to generate ROIs in BVs for analysis. We found that the number of CALR punctae in AQP4 co-stained endfeet decreased by $\sim 2 \%$ from $250.23 \pm 17.6$ per BV in young mice to $147.28 \pm 8.23$ per $\mathrm{BV}$ in aging mice (Figure $6 \mathrm{~B}$ ). In addition, when compared to young mice, CALR intensity in endfeet was reduced by $\sim 20 \%$ (Figure 6C). This reduction in CALR intensity in aging mice was not restricted to endfeet since we observed a general $15 \%$ decrease in CALR intensity throughout the neuropil of the DLS in aging mice (Figure 6D). Thus, when compared to young mice, aging mice demonstrate a significant reduction in expression of the major $\mathrm{Ca}^{2+}$ buffer, CALR within the neuropil and astrocytic endfeet of the DLS.

\section{Aging increases the frequency of spontaneous $\mathrm{Ca}^{2+}$ influx into endfoot mitochondria}

Since the ER serves as a major source for $\mathrm{Ca}^{2+}$ influx into astrocytic mitochondria in the DLS (Huntington and Srinivasan, 2021), we rationalized that an aging-related reduction of CALR within astrocytic endfeet would likely alter $\mathrm{Ca}^{2+}$ influx into endfoot mitochondria.

To specifically measure $\mathrm{Ca}^{2+}$ influx into endfoot mitochondria in the DLS, young and aging mice were stereotaxically injected with a previously described construct, AAV2/5- 
GfaABC1D-mito7-GCaMP6f (Huntington and Srinivasan, 2021), and confocal imaging was performed in live striatal brain slices from young and aging mice with TL as a marker for BVs (Figure 7A and B). Endfoot mitochondria in young and aging mice displayed robust spontaneous $\mathrm{Ca}^{2+}$ influx events (Figure 7B and C; supplementary movies 5 and 6). However, when compared to young mice, aging mice showed a $35 \%$ decrease in the number of endfoot mitochondrial $\mathrm{Ca}^{2+}$ event ROIs and a $~ 50 \%$ increase in the size of ROIs (Figure 7D).

There were also significant aging-related changes in the kinetics of mitochondrial $\mathrm{Ca}^{2+}$ influx events. When compared to young mice, aging mice demonstrated a dramatic $50 \%$ increase in the frequency of endfoot mitochondrial $\mathrm{Ca}^{2+}$ influx (Figure 8A). Cumulative frequency distributions of DLS endfoot $\mathrm{Ca}^{2+}$ events showed that when compared to young mice with an equal distribution of mitochondrial $\mathrm{Ca}^{2+}$ influx across the entire frequency range $(0.2$ to 2.0 events/min), the vast majority ( 93\%) of $\mathrm{Ca}^{2+}$ events in aging mice occurred at a significantly higher range (0.2 to 2.4 events/min) (Figure $8 \mathrm{~B})$. Despite these changes, the interval between independent endfoot mitochondrial $\mathrm{Ca}^{2+}$ events across BVs from multiple mice were similar in young and aging mice (Figure 8C). For both young and aging mice, there were no significant alterations in the amplitude or half width of spontaneous mitochondrial $\mathrm{Ca}^{2+}$ influx events (Figure 8D). Thus, aging-related increases in $\mathrm{ER} \mathrm{Ca}^{2+}$ specifically caused a dramatic increase in the frequency of $\mathrm{Ca}^{2+}$ influx in endfoot mitochondria of the DLS.

\section{$\mathrm{Ca}^{2+}$ influx into endfoot mitochondria of the DLS in aging mice requires $\mathrm{ER} \mathrm{Ca} \mathrm{Ca}^{2+}$ stores}

The observed increase in $\mathrm{ER} \mathrm{Ca}^{2+}$ stores within astrocytic endfeet of aging mice, along with a significant aging-related increase in the frequency of $\mathrm{Ca}^{2+}$ influx into endfoot mitochondria led us to ask if $\mathrm{ER}^{2+2}$ stores are a primary source for $\mathrm{Ca}^{2+}$ influx into astrocytic endfoot mitochondria for both young and aging mice. To test this idea, we sequentially depleted 
extracellular, followed by intracellular $\mathrm{Ca}^{2+}$ sources in DLS slices. In each case, we measured the kinetics of $\mathrm{Ca}^{2+}$ influx into endfoot mitochondria within the DLS of young and aging mice. Extracellular $\mathrm{Ca}^{2+}$ depletion with zero $\mathrm{Ca}^{2+} \mathrm{aCSF}$ did not alter the amplitude of endfoot mitochondrial $\mathrm{Ca}^{2+}$ influx in young and aging mice (Figure 9B and D; supplementary movies 5 and 6). Young mice demonstrated no change in $\mathrm{Ca}^{2+}$ event frequency and a small decrease in the half width of mitochondrial $\mathrm{Ca}^{2+}$ signals (Figure 9B). For aging mice, we observed a small decrease in the frequency, but no change in the half width of mitochondrial $\mathrm{Ca}^{2+}$ signals (Figure

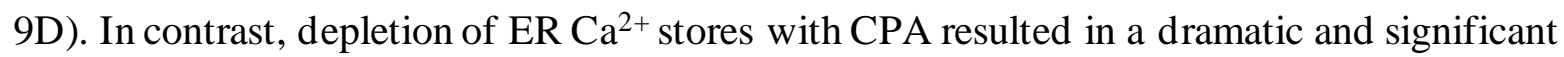
$\sim 80 \%$ decrease in the frequency, amplitude and half width of mitochondrial end foot $\mathrm{Ca}^{2+}$ influx in young mice (Figure 10A and B; supplementary movies 7 and 8). In aging mice, we observed a significant $77 \%$ decrease in frequency, $65 \%$ decrease in amplitude, and 59\% decrease in event half width (Figure 10C and D). These data confirm that the ER is indeed a primary source for $\mathrm{Ca}^{2+}$ influx into astrocytic endfoot mitochondria for both young and aging mice.

\section{Discussion}

Aging is associated with subtle changes in neuronal and astrocytic $\mathrm{Ca}^{2+}$ homeostasis (Guerra-Gomes et al., 2018; Nikoletopoulou and Tavernarakis, 2012; Toescu and Verkhratsky, 2007). In addition, neurovascular impairment is known to initiate neurodegeneration (Cabezas et al., 2014; Cai et al., 2017; Govindpani et al., 2019; Grammas et al., 1999; Ott et al., 2018; Sweeney et al., 2019; Yu et al., 2020; Zhou et al., 2020), and aging alters astrocytic gene expression (Boisvert et al., 2018). These seemingly unrelated findings point to the idea that aging could alter $\mathrm{Ca}^{2+}$-mediated coupling between astrocytic endfeet and the neurovasculature, eventually leading to neurodegeneration. In this context, we asked if astrocytic endfeet in the 
DLS of aging mice display changes in expression of the $\mathrm{Ca}^{2+}$ buffering protein, CALR, and alterations in the sources and kinetics of astrocytic endfoot $\mathrm{Ca}^{2+}$ signals.

We found that aging astrocytic endfeet in the DLS demonstrate a significant reduction in CALR (Figure 6), which is the primary endogenous buffer for ER-localized $\mathrm{Ca}^{2+}$. This reduction in CALR is associated with alterations in multiple aspects of spontaneous cytosolic and mitochondrial $\mathrm{Ca}^{2+}$ events within astrocytic endfeet (Figures 1,2,3,7 and 8). Based on these data, we infer that the aging-related reduction of CALR expression is upstream of alterations in endfoot $\mathrm{Ca}^{2+}$ signals. Multiple lines of evidence support our interpretation of the data:(i) Astrocytic endfeet contain ER and mitochondria (Boulay et al., 2017; Gobel et al., 2020), (ii) The generation of spontaneous endfoot $\mathrm{Ca}^{2+}$ signals in aging mice exclusively depends on ER rather than extracellular $\mathrm{Ca}^{2+}$ stores (Figure 4C and F), and (iii) As previously shown by Biwer et $a l$, conditional deletion of CALR from end othelial cells in aging mice increases the spatial spread of spontaneous endothelial $\mathrm{Ca}^{2+}$ signals and impairs $\mathrm{Ca}^{2+}$ mobilization (Biwer et al., 2020).

Similar to the Biwer et al study which was conducted in mice with a conditional knockout of CALR in endothelial cells (Biwer et al., 2020), the current study shows that reduced CALR expression in the astrocytic endfeet of aging mice is associated with an increase in the velocity of expansion of DLS endfoot $\mathrm{Ca}^{2+}$ signals (Figure 3). Since CALR is known to regulate $\mathrm{Ca}^{2+}$ mobilization within the ER (Nakamura et al., 2001), our findings suggest that the observed changes in $\mathrm{Ca}^{2+}$ signal kinetics likely stem from the aging-related reduction of CALR within astrocytic endfeet. In accordance with this rationale, we observed very specific aging-related changes in the kinetics of cytosolic astrocytic endfoot $\mathrm{Ca}^{2+}$ signals. There was an increase in the 
number of low frequency endfoot $\mathrm{Ca}^{2+}$ signals in aging mice (Figure 2B) accompanied by a nearly 2-fold increase in the amplitude as well as an increase in the duration of $\mathrm{Ca}^{2+}$ signals (Figure 2D). These data suggest that aging mice possess a generalized dysregulation of $\mathrm{Ca}^{2+}$ signaling in astrocytic endfeet of the DLS.

Our finding that endfoot $\mathrm{Ca}^{2+}$ signals in aging mice exclusively depend on ER stores (Figure 4F) strongly suggests that the observed changes in cytosolic $\mathrm{Ca}^{2+}$ signal kinetics likely reflect $\mathrm{Ca}^{2+}$ efflux from the ER into the cytosol of astrocytic endfeet. The ER in astrocytic endfeet is morphologically and functionally coupled with mitochondria (Boulay et al., 2017; Gobel et al., 2020), enabling mitochondria to sequester $\mathrm{Ca}^{2+}$ released from the ER. Therefore, one downstream target of aging-related alterations in $\mathrm{Ca}^{2+}$ efflux from the ER of astrocytic endfeet would be changes in mitochondrial $\mathrm{Ca}^{2+}$ signals. In line with this rationale, we showed that when compared to young mice, aging-related alterations in endfoot $\mathrm{Ca}^{2+}$ efflux from the ER of aging mice was associated with a concomitant increase in the frequency of $\mathrm{Ca}^{2+}$ influx events into end foot mitochondria (Figure $8 \mathrm{~A}$ and $\mathrm{B}$ ). The finding that mitochondrial $\mathrm{Ca}^{2+}$ influx increases in frequency with a corresponding decrease in the frequency of cytosolic $\mathrm{Ca}^{2+}$ signals suggests a general aging-related dysregulation of functional coupling between the ER and mitochondria in astrocytic endfeet of the DLS. Given the importance of normal AQP4 localization and function in maintaining BBB integrity (Vella et al., 2015), one downstream consequence of reduced CALR expression and altered $\mathrm{Ca}^{2+}$ signals in aging astrocytic endfeet may be the gradual loss of AQP4, resulting in a loss of BBB integrity and a consequent increase in the risk for neurodegeneration. In support of this idea, a reduction in CALR expression has been linked to the loss of motor neurons during amyotrophic lateral sclerosis (ALS) (BernardMarissal et al., 2012), and physical and functional changes in the NVU have been established as 
an important contributor to neurodegenerative disorders such as AD and PD (Cabezas et al., 2014; Cai et al., 2017; Govindpani et al., 2019; Grammas et al., 1999; Ott et al., 2018; Sweeney et al., 2019; Yu et al., 2020; Zhou et al., 2020). Apart from altering BBB integrity, subtle changes in the localization of $\mathrm{Ca}^{2+}$ fluxes within the aging astrocytic endfoot could have important consequences for vascular end othelial cell function. Conceivably, altered $\mathrm{Ca}^{2+}$ signals could change the activation of large-conductance $\mathrm{Ca}^{2+}$ activated $\mathrm{K}^{+}(\mathrm{BK})$ channels in aging endfeet (Masamoto et al., 2015), thereby disrupting $\mathrm{K}^{+}$-dependent endothelial cell-mediated capillary to arteriole ascending dilation within the brain (Bagher and Segal, 2011; Longden et al., 2014; Longden et al., 2017).

In summary, we show that an aging-related reduction in CALR expression within astrocytic endfeet results in the exclusive dependence of endfoot $\mathrm{Ca}^{2+}$ signals on $\mathrm{ER} \mathrm{Ca}^{2+}$, which results in abnormal $\mathrm{Ca}^{2+}$ coupling between the ER and mitochondria. Based on our data, we propose that altered $\mathrm{Ca}^{2+}$ buffering and aging-related dysregulation in endfoot $\mathrm{Ca}^{2+}$ signaling could conceivably alter the localization of AQP4 within astrocytic endfeet, thereby compromising BBB integrity, as well as NVU coupling. Taken together, our findings have important implications for understanding how BBB and NVU dysfunction in the aging brain can trigger, and possibly sustain neurodegenerative processes.

\section{References}

Attwell, D., Buchan, A.M., Charpak, S., Lauritzen, M., Macvicar, B.A., Newman, E.A., 2010. Glial and neuronal control of brain blood flow. Nature 468(7321), 232-243.

Bagher, P., Segal, S.S., 2011. Regulation of blood flow in the microcirculation: role of conducted vasodilation. Acta Physiol (Oxf) 202(3), 271-284. 
Belanger, M., Allaman, I., Magistretti, P.J., 2011. Brain energy metabolism: focus on astrocyteneuron metabolic cooperation. Cell Metab 14(6), 724-738.

Bernard-Marissal, N., Moumen, A., Sunyach, C., Pellegrino, C., Dudley, K., Henderson, C.E., Raoul, C., Pettmann, B., 2012. Reduced calreticulin levels link endoplasmic reticulum stress and Fas-triggered cell death in motoneurons vulnerable to ALS. J Neurosci 32(14), 4901-4912.

Biwer, L.A., Askew-Page, H.R., Hong, K., Milstein, J., Johnstone, S.R., Macal, E., Good, M.E., Bagher, P., Sonkusare, S.K., Isakson, B.E., 2020. Endothelial calreticulin deletion impairs endothelial function in aged mice. Am J Physiol Heart Circ Physiol 318(5), H1041-H1048.

Boisvert, M.M., Erikson, G.A., Shokhirev, M.N., Allen, N.J., 2018. The Aging Astrocyte Transcriptome from Multiple Regions of the Mouse Brain. Cell Rep 22(1), 269-285.

Boulay, A.C., Saubamea, B., Adam, N., Chasseigneaux, S., Mazare, N., Gilbert, A., Bahin, M., Bastianelli, L., Blugeon, C., Perrin, S., Pouch, J., Ducos, B., Le Crom, S., Genovesio, A., Chretien, F., Decleves, X., Laplanche, J.L., Cohen-Salmon, M., 2017. Translation in astrocyte distal processes sets molecular heterogeneity at the gliovascular interface. Cell Discov 3, 17005. Cabezas, R., Avila, M., Gonzalez, J., El-Bacha, R.S., Baez, E., Garcia-Segura, L.M., Jurado Coronel, J.C., Capani, F., Card ona-Gomez, G.P., Barreto, G.E., 2014. Astrocytic modulation of blood brain barrier: perspectives on Parkinson's disease. Front Cell Neurosci 8, 211.

Cai, W., Zhang, K., Li, P., Zhu, L., Xu, J., Yang, B., Hu, X., Lu, Z., Chen, J., 2017. Dysfunction of the neurovascular unit in ischemic stroke and neurodegenerative diseases: An aging effect. Ageing Res Rev 34, 77-87.

Chung, S.J., Yoo, H.S., Shin, N.Y., Park, Y.W., Lee, H.S., Hong, J.M., Kim, Y.J., Lee, S.K., Lee, P.H., Sohn, Y.H., 2021. Perivascular Spaces in the Basal Ganglia and Long-term Motor Prognosis in Newly Diagnosed Parkinson Disease. Neurology 96(16), e2121-e2131. 
Duda, J.E., Giasson, B.I., Mabon, M.E., Lee, V.M., Trojanowski, J.Q., 2002. Novel antibodies to synuclein show abundant striatal pathology in Lewy body diseases. Ann Neurol 52(2), 205-210.

Dunn, K.M., Hill-Eubanks, D.C., Liedtke, W.B., Nelson, M.T., 2013. TRPV4 channels stimulate $\mathrm{Ca} 2+$-induced $\mathrm{Ca} 2+$ release in astrocytic endfeet and amplify neurovascular coupling responses. Proc Natl Acad Sci U S A 110(15), 6157-6162.

Eilert-Olsen, M., Hjukse, J.B., Thoren, A.E., Tang, W., Enger, R., Jensen, V., Pettersen, K.H., Nagelhus, E.A., 2019. Astroglial endfeet exhibit distinct $\mathrm{Ca}(2+)$ signals during hypoosmotic conditions. Glia 67(12), 2399-2409.

Feekes, J.A., Cassell, M.D., 2006. The vascular supply of the functional compartments of the human striatum. Brain 129(Pt 8), 2189-2201.

Feekes, J.A., Hsu, S.W., Chaloupka, J.C., Cassell, M.D., 2005. Tertiary microvascular territories define lacunar infarcts in the basal ganglia. Ann Neurol 58(1), 18-30.

Garcia, D.C.G., Longden, T.A., 2020. Ion channels in capillary endothelium. Curr Top Membr 85, 261-300.

Girouard, H., Bonev, A.D., Hannah, R.M., Meredith, A., Aldrich, R.W., Nelson, M.T., 2010. Astrocytic endfoot $\mathrm{Ca} 2+$ and $\mathrm{BK}$ channels determine both arteriolar dilation and constriction. Proc Natl Acad Sci U S A 107(8), 3811-3816.

Gobel, J., Engelhardt, E., Pelzer, P., Sakthivelu, V., Jahn, H.M., Jevtic, M., Folz-Donahue, K., Kukat, C., Schauss, A., Frese, C.K., Giavalisco, P., Ghanem, A., Conzelmann, K.K., Motori, E., Bergami, M., 2020. Mitochondria-Endoplasmic Reticulum Contacts in Reactive Astrocytes Promote Vascular Remodeling. Cell Metab 31(4), 791-808 e798. 
Govindpani, K., McNamara, L.G., Smith, N.R., Vinnakota, C., Waldvogel, H.J., Faull, R.L., Kwakowsky, A., 2019. Vascular Dysfunction in Alzheimer's Disease: A Prelude to the Pathological Process or a Consequence of It? J Clin Med 8(5).

Grammas, P., Moore, P., Weigel, P.H., 1999. Microvessels from Alzheimer's disease brains kill neurons in vitro. Am J Pathol 154(2), 337-342.

Gray, M.T., Woulfe, J.M., 2015. Striatal blood-brain barrier permeability in Parkinson's disease. J Cereb Blood Flow Metab 35(5), 747-750.

Guerra-Gomes, S., Viana, J.F., Nascimento, D.S.M., Correia, J.S., Sardinha, V.M., Caetano, I., Sousa, N., Pinto, L., Oliveira, J.F., 2018. The Role of Astrocytic Calcium Signaling in the Aged Prefrontal Cortex. Front Cell Neurosci 12, 379.

Haley, M.J., Lawrence, C.B., 2017. The blood-brain barrier after stroke: Structural studies and the role of transcytotic vesicles. J Cereb Blood Flow Metab 37(2), 456-470.

Hanseeuw, B.J., Lopera, F., Sperling, R.A., Norton, D.J., Guzman-Velez, E., Baena, A., PardillaDelgado, E., Schultz, A.P., Gatchel, J., Jin, D., Chen, K., Reiman, E.M., Johnson, K.A., Quiroz, Y.T., 2019. Striatal amyloid is associated with tauopathy and memory decline in familial Alzheimer's disease. Alzheimers Res Ther 11(1), 17.

Heithoff, B.P., George, K.K., Phares, A.N., Zuidhoek, I.A., Munoz-Ballester, C., Robel, S., 2021. Astrocytes are necessary for blood-brain barrier maintenance in the adult mouse brain. Glia 69(2), 436-472.

Hou, Y., Dan, X., Babbar, M., Wei, Y., Hasselbalch, S.G., Croteau, D.L., Bohr, V.A., 2019. Ageing as a risk factor for neurodegenerative disease. Nat Rev Neurol 15(10), 565-581.

Howarth, C., 2014. The contribution of astrocytes to the regulation of cerebral blood flow. Front Neurosci 8, 103. 
Huntington, T.E., Srinivasan, R., 2021. Astrocytic mitochondria in adult mouse brain slices show spontaneous calcium influx events with unique properties. Cell Calcium 96, 102383.

Irwin, K., Sexton, C., Daniel, T., Lawlor, B., Naci, L., 2018. Healthy Aging and Dementia: Two Roads Diverging in Midlife? Front Aging Neurosci 10, 275.

Ivanidze, J., Skafida, M., Pandya, S., Patel, D., Osborne, J.R., Raj, A., Gupta, A., Henchcliffe, C., Dyke, J.P., 2020. Molecular Imaging of Striatal Dopaminergic Neuronal Loss and the Neurovascular Unit in Parkinson Disease. Front Neurosci 14, 528809.

Jiang, R., Haustein, M.D., Sofroniew, M.V., Khakh, B.S., 2014. Imaging intracellular Ca(2)(+) signals in striatal astrocytes from adult mice using genetically-encoded calcium indicators. $\mathrm{J}$ Vis $\operatorname{Exp}(93)$, e51972.

Leritz, E.C., McGlinchey, R.E., Kellison, I., Rudolph, J.L., Milberg, W.P., 2011. Cardiovascular Disease Risk Factors and Cognition in the Elderly. Curr Cardiovasc Risk Rep 5(5), 407-412. Longden, T.A., Dabertrand, F., Hill-Eubanks, D.C., Hammack, S.E., Nelson, M.T., 2014. Stressinduced glucocorticoid signaling remodels neurovascular coupling through impairment of cerebrovascular inwardly rectifying K+ channel function. Proc Natl Acad Sci U S A 111(20), $7462-7467$.

Longden, T.A., Dabertrand, F., Koide, M., Gonzales, A.L., Tykocki, N.R., Brayden, J.E., HillEubanks, D., Nelson, M.T., 2017. Capillary K(+)-sensing initiates retrograde hyperpolarization to increase local cerebral blood flow. Nat Neurosci 20(5), 717-726.

Masamoto, K., Unekawa, M., Watanabe, T., Toriumi, H., Takuwa, H., Kawaguchi, H., Kanno, I., Matsui, K., Tanaka, K.F., Tomita, Y., Suzuki, N., 2015. Unveiling astrocytic control of cerebral blood flow with optogenetics. Sci Rep 5, 11455. 
Mishra, A., Reynolds, J.P., Chen, Y., Gourine, A.V., Rusakov, D.A., Attwell, D., 2016.

Astrocytes mediate neurovascular signaling to capillary pericytes but not to arterioles. Nat Neurosci 19(12), 1619-1627.

Nakamura, K., Zuppini, A., Arnaudeau, S., Lynch, J., Ahsan, I., Krause, R., Papp, S., De Smedt, H., Parys, J.B., Muller-Esterl, W., Lew, D.P., Krause, K.H., Demaurex, N., Opas, M., Michalak, M., 2001. Functional specialization of calreticulin domains. J Cell Biol 154(5), 961-972.

Nikoletopoulou, V., Tavernarakis, N., 2012. Calcium homeostasis in aging neurons. Front Genet 3, 200.

Ott, B.R., Jones, R.N., Daiello, L.A., de la Monte, S.M., Stopa, E.G., Johanson, C.E., Denby, C., Grammas, P., 2018. Blood-Cerebrospinal Fluid Barrier Gradients in Mild Cognitive Impairment and Alzheimer's Disease: Relationship to Inflammatory Cytokines and Chemokines. Front Aging Neurosci 10, 245.

Pandit, R., Chen, L., Gotz, J., 2020. The blood-brain barrier: Physiology and strategies for drug delivery. Adv Drug Deliv Rev 165-166, 1-14.

Rosso, A.L., Bohnen, N.I., Launer, L.J., Aizenstein, H.J., Yaffe, K., Rosano, C., 2018. Vascular and dopaminergic contributors to mild parkinsonian signs in older adults. Neurology 90(3), e223-e229.

Srinivasan, R., Huang, B.S., Venugopal, S., Johnston, A.D., Chai, H., Zeng, H., Golshani, P., Khakh, B.S., 2015. Ca(2+) signaling in astrocytes from Ip3r2(-/-) mice in brain slices and during startle responses in vivo. Nat Neurosci 18(5), 708-717.

Srinivasan, R., Lu, T.Y., Chai, H., Xu, J., Huang, B.S., Golshani, P., Coppola, G., Khakh, B.S., 2016. New Transgenic Mouse Lines for Selectively Targeting Astrocytes and Studying Calcium Signals in Astrocyte Processes In Situ and In Vivo. Neuron 92(6), 1181-1195. 
Sweeney, M.D., Sagare, A.P., Zlokovic, B.V., 2018. Blood-brain barrier breakdown in

Alzheimer disease and other neurodegenerative disorders. Nat Rev Neurol 14(3), 133-150.

Sweeney, M.D., Zhao, Z., Montagne, A., Nelson, A.R., Zlokovic, B.V., 2019. Blood-Brain

Barrier: From Physiology to Disease and Back. Physiol Rev 99(1), 21-78.

Toescu, E.C., Verkhratsky, A., 2007. The importance of being subtle: small changes in calcium homeostasis control cognitive decline in normal aging. Aging Cell 6(3), 267-273.

Vella, J., Zammit, C., Di Giovanni, G., Muscat, R., Valentino, M., 2015. The central role of aquaporins in the pathophysiology of ischemic stroke. Front Cell Neurosci 9, 108.

Villabona-Rueda, A., Erice, C., Pardo, C.A., Stins, M.F., 2019. The Evolving Concept of the Blood Brain Barrier (BBB): From a Single Static Barrier to a Heterogeneous and Dynamic Relay Center. Front Cell Neurosci 13, 405.

Yousufuddin, M., Young, N., 2019. Aging and ischemic stroke. Aging (Albany NY) 11(9), 2542-2544.

Yu, X., Ji, C., Shao, A., 2020. Neurovascular Unit Dysfunction and Neurodegenerative Disorders. Front Neurosci 14, 334.

Zhang, C., Tabatabaei, M., Belanger, S., Girouard, H., Moeini, M., Lu, X., Lesage, F., 2019. Astrocytic endfoot $\mathrm{Ca}(2+)$ correlates with parenchymal vessel responses during 4-AP induced epilepsy: An in vivo two-photon lifetime microscopy study. J Cereb Blood Flow Metab 39(2), 260-271.

Zhou, Y., Chen, Q., Wang, Y., Wu, H., Xu, W., Pan, Y., Gao, S., Dong, X., Zhang, J.H., Shao, A., 2020. Persistent Neurovascular Unit Dysfunction: Pathophysiological Substrate and Trigger for Late-Onset Neurodegeneration After Traumatic Brain Injury. Front Neurosci 14, 581. 


\section{Figure Legends}

Figure 1. Astrocyte endfoot expression of Lck-GCaMP6f in young and aging mice. (A) AAV-GfaABC1D-Lck-GCaMP6f was injected into the DLS of young and aging mice. 2 weeks later, intraventricular injection of tomato lectin (TL) was performed, and striatal brain sections were collected for recording and measuring endfoot $\mathrm{Ca}^{2+}$ events in the DLS. (B) Representative images of young and aging astrocyte endfeet expressing Lck-GCaMP6f (green) immediately adjacent to TL labeled blood vessels (red) in the DLS, scale bar $=15 \mu \mathrm{m}$. White arrows point to areas where endfoot $\mathrm{Ca}^{2+}$ events initiated. (C) Example traces of endfoot $\mathrm{Ca}^{2+}$ events recorded from young and aging mice. (D) Comparisons of the number (left) and area (right) of ROIs generated in young and aging mice. For young mice, $n=64$ ROIs and 20 blood vessels from 5 mice. For aging mice, $\mathrm{n}=57$ ROIs and 26 blood vessels from 7 mice. Error bars are S.E.M and all $p$ values are based on Mann-Whitney test.

Figure 2. Aging alters $\mathrm{Ca}^{2+}$ event kinetics. (A) Plot of $\mathrm{Ca}^{2+}$ event frequencies in the endfeet of young and aging mice (B) Cumulative distribution of frequencies in young and aging mice (C) Plot of frequency intervals in young and aging mice (D) Amplitude (left) and half width (right) of spontaneous $\mathrm{Ca}^{2+}$ events in young and aging mice. For young mice, $\mathrm{n}=64 \mathrm{ROIs}$ and 20 blood vessels from 5 mice. For aged mice, $n=57$ ROIs and 26 blood vessels from 7 mice. Error bars are S.E.M and for panels A, C, and D $p$ values are based on Mann-Whitney tests. For the cumulative frequency plot in panel $\mathrm{B}$, the $p$ value is based on a Kolmogorov-Smirnov test.

Figure 3. Aging increases the velocity of expanding $\mathrm{Ca}^{2+}$ waves in astrocyte endfeet. (A) A representative t-stack of an expanding endfoot $\mathrm{Ca}^{2+}$ wave in the DLS of a young (top) and aging (bottom) mouse is shown. Panels to the right of t-stacks show representative pseudo-colored time 
lapse images of endfoot $\mathrm{Ca}^{2+}$ waves for young (top) and aging (bottom) mice. The white dotted lines in time-lapse images outline the maximum area attained by expanding endfoot $\mathrm{Ca}^{2+}$ waves, scale bar $=15 \mu \mathrm{m}(\mathbf{B})$ Bar graphs showing the velocity (left), distance traveled (middle), and area (right) of endfoot $\mathrm{Ca}^{2+}$ waves. For young mice, $\mathrm{n}=14$ ROIs and 6 blood vessels from 3 mice. For aging mice, $\mathrm{n}=9$ ROIs and 4 blood vessels from 3 mice Error bars are S.E.M and all $p$ values are based on Mann-Whitney tests.

Figure 4. Astrocyte endfeet in aging mice exclusively rely on $\mathrm{ER} \mathrm{Ca}^{2+}$ stores to generate spontaneous $\mathrm{Ca}^{2+}$ events. (A) Representative endfoot $\mathrm{Ca}^{2+}$ event traces from the DLS of young mice after bath application of zero $\mathrm{Ca}^{2+}$ aCSF or after 15 min incubation with $20 \mu \mathrm{M}$ CPA (B) Bar graphs showing the average $\mathrm{Ca}^{2+}$ event frequency for young mice after bath application of zero $\mathrm{Ca}^{2+}$ aCSF (left graph) or after 15 min incubation with $20 \mu \mathrm{M} \mathrm{CPA}$ (right graph) (C) Bar graph of average \% decrease in $\mathrm{Ca}^{2+}$ event frequency in young mice exposed to either zero $\mathrm{Ca}^{2+}$ aCSF or CPA (D) Representative endfoot $\mathrm{Ca}^{2+}$ event traces from the DLS of aging mice after bath application of zero $\mathrm{Ca}^{2+}$ aCSF or after 15 min incubation with $20 \mu \mathrm{M}$ CPA (E) Bar graphs showing the average $\mathrm{Ca}^{2+}$ event frequency for aging mice after bath application of zero $\mathrm{Ca}^{2+}$ aCSF (left graph) or after 15 min incubation with $20 \mu \mathrm{M} \mathrm{CPA}$ (right graph) (F) Bar graph of average \% decrease in $\mathrm{Ca}^{2+}$ event frequency in aging mice exposed to either zero $\mathrm{Ca}^{2+}$ aCSF or CPA. For zero $\mathrm{Ca}^{2+}$ in young mice, $\mathrm{n}=64$ ROIs and 20 blood vessels from 5 mice. For zero $\mathrm{Ca}^{2+}$ in aging mice, $\mathrm{n}=57$ ROIs and 26 blood vessels from 7 mice. For CPA experiments in young mice, $\mathrm{n}=21$ ROIs and 11 blood vessels from 5 mice. For CPA experiments in aging mice, $\mathrm{n}=$ 24 ROIs and 11 blood vessels from 6 mice Error bars are S.E.M and $p$ values are based on Wilcoxon signed ranked tests. 
Figure 5. CPA alters endfoot $\mathrm{Ca}^{2+}$ event amplitude and duration in young and aging mice.

(A) Bar graphs showing amplitude (left) and half width (right) for $\mathrm{Ca}^{2+}$ events with zero $\mathrm{Ca}^{2+}$ in young mice (B) Bar graphs showing amplitude (left) and half width (right) for $\mathrm{Ca}^{2+}$ events with zero $\mathrm{Ca}^{2+}$ in aging mice $(\mathbf{C})$ Bar graphs showing amplitude (left) and half width (right) for $\mathrm{Ca}^{2+}$ events with CPA in young mice (D) Bar graphs showing amplitude (left) and half width (right) for $\mathrm{Ca}^{2+}$ events with CPA in aging mice. For zero $\mathrm{Ca}^{2+}$ experiments, young mice, $\mathrm{n}=64$ ROIs and 20 blood vessels from 5 mice. For aging mice, $\mathrm{n}=57$ ROIs and 26 blood vessels from 7 mice. For CPA experiments, young mice, $\mathrm{n}=21$ ROIs and 11 blood vessels from 5 mice and aging mice, $\mathrm{n}=24$ ROIs and 11 blood vessels from 6 mice. Error bars are S.E.M and $p$ values are based on Wilcoxon signed ranked tests.

Figure 6. Aging reduces CALR expression levels in astrocyte endfeet and in the neuropil of the DLS. (A) Z-stack of representative confocal images from the DLS of young (left) and aged (right) mice co-labeled with AQP4 (green) and CALR (red), scale bar $=15 \mu \mathrm{m}$. The white arrows point to individual CALR punctae that colocalize with AQP4 staining. (B) Bar graph showing the number of CALR punctae in the DLS astrocytic endfeet of young and aging mice (C) Bar graph showing CALR intensity within AQP4 labeled BVs of young and aging mice (D) Bar graph comparing CALR intensity in the entire neuropil of the DLS from young and aging mouse brain sections. For young and aging mice $n=18$ blood vessels from 3 striatal sections per mouse and 3 mice per group. Error bars are S.E.M, $p$ values are based on MannWhitney tests.

Figure 7. Astrocyte endfoot expression of mito7-GCaMP6f in young and aging mice. (A) AAV-GfaABC1D-mito7-GCaMP6f was injected into the DLS of young and aging mice. 3 weeks later, intraventricular injection of tomato lectin (TL) was performed, and striatal brain 
sections were collected for recording and measuring $\mathrm{Ca}^{2+}$ influx events in endfoot mitochondria within the DLS. (B) Representative t-stacks of mitochondrial $\mathrm{Ca}^{2+}$ influx events detected by mito7-GCaMP6f (green) in young and aging astrocyte endfeet immediately adjacent to TL labeled blood vessels (red) in the DLS, scale bar $=15 \mu \mathrm{m}$. White arrows point to areas where mitochondrial endfoot $\mathrm{Ca}^{2+}$ events initiated. (C) Representative endfoot mitochondrial $\mathrm{Ca}^{2+}$ influx event traces from young (red) and aging (blue) mice. (D) Bar graphs showing the number (left) and area (right) of endfoot mitochondrial $\mathrm{Ca}^{2+}$ event ROIs in young and aging mice. For young mice, $\mathrm{n}=52$ ROIs and 18 blood vessels from 3 mice. For aging mice, $\mathrm{n}=28$ ROIs and 16 blood vessels from 5 mice. Error bars are S.E.M and all $p$ values are based on Mann-Whitney tests.

Figure 8. Aging increases the frequency of endfoot mitochondrial $\mathrm{Ca}^{2+}$ events. (A) Bar graph of mitochond rial $\mathrm{Ca}^{2+}$ event frequencies in the endfeet of young and aging mice (B) Cumulative distribution of mitochondrial $\mathrm{Ca}^{2+}$ event frequencies in young and aging mice $(\mathbf{C})$ Plot of mitochondrial frequency intervals in young and aging mice (D) Amplitude (left) and half width (right) of mitochondrial $\mathrm{Ca}^{2+}$ events in young and aging mice. For young mice, $\mathrm{n}=64$ ROIs and 20 blood vessels from 5 mice. For young mice, $n=52$ ROIs and 18 blood vessels from 3 mice. For aging mice, $\mathrm{n}=28$ ROIs and 16 blood vessels from 5 mice. Error bars are S.E.M and $p$ values in panels A, C, and D are based on Mann-Whitney tests. For the cumulative frequency plot in panel $\mathrm{B}$, the $p$ value is based on a Kolmogorov-Smirnov test.

Figure 9. Endfoot mitochondria in young and aging mice do not rely on extracellular $\mathrm{Ca}^{2+}$. (A) Representative mitochondrial $\mathrm{Ca}^{2+}$ event traces from young mice before and after bath application of zero $\mathrm{Ca}^{2+} \mathrm{aCSF}$ (B) Bar graphs showing frequency (left), amplitude (middle), and half width (right) for mitochondrial $\mathrm{Ca}^{2+}$ events recorded in young mice with and without zero 
$\mathrm{Ca}^{2+} \mathrm{aCSF}(\mathbf{C})$ Representative mitochondrial $\mathrm{Ca}^{2+}$ event traces from aging mice before and after bath application of zero $\mathrm{Ca}^{2+} \mathrm{aCSF}(\mathbf{D})$ Bar graphs showing frequency (left), amplitude (middle), and halfwidth (right) for mitochondrial $\mathrm{Ca}^{2+}$ events recorded in aging mice with and without zero $\mathrm{Ca}^{2+} \mathrm{aCSF}$. For young mice, $\mathrm{n}=52$ ROIs and 18 blood vessels from 3 mice. For aging mice, $\mathrm{n}=$ 28 ROIs and 16 blood vessels from 5 mice. Error bars are S.E.M and all $p$ values are based on Wilcoxon signed ranked tests.

Figure 10. Endfoot mitochondria in young and aging mice depend on intracellular $\mathrm{Ca}^{2+}$ stores. (A) Representative mitochondrial $\mathrm{Ca}^{2+}$ event traces from young mice before and after bath application of CPA (B) Bar graphs showing frequency (left), amplitude (middle), and half width (right) for mitochondrial $\mathrm{Ca}^{2+}$ events recorded in young mice with and without $\mathrm{CPA}$ (C) Representative mitochondrial $\mathrm{Ca}^{2+}$ event traces from aging mice before and after bath application of CPA (D) Bar graphs showing frequency (left), amplitude (middle), and halfwidth (right) for mitochond rial $\mathrm{Ca}^{2+}$ events recorded in aging mice with and without CPA. For young mice, $\mathrm{n}=22$ ROIs and 9 blood vessels from 3 mice. For aging mice, $\mathrm{n}=18$ ROIs and 11 blood vessels from 5 mice All errors are SEM; $p$ values are based on Wilcoxon signed ranked tests. 

(which was not certified by peer review) is the author/funder, who has granted bioRxiv a license to display the preprint in perpetuity. It is made
available under aCC-BY-NC-ND 4.0 International license. Figure 1

A Injection of AAV into DLS

GfaAB1D-Lck GCaMP6f

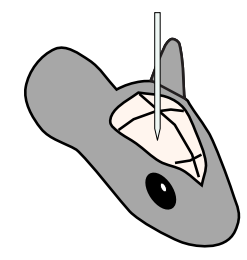

Young:

3-4 months old 20-24 months old
Confocal Imaging

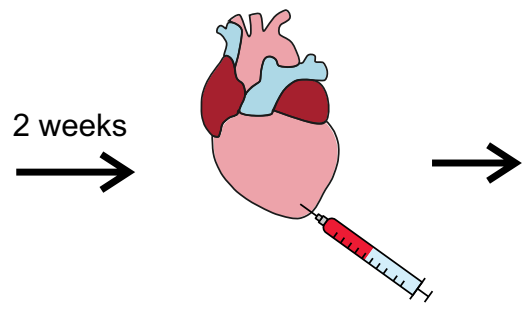

Intraventricular injection of $T L$

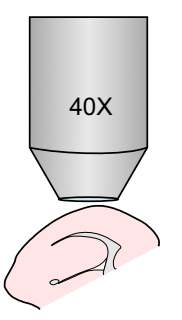

Imaging of striatal brain slice
Analysis

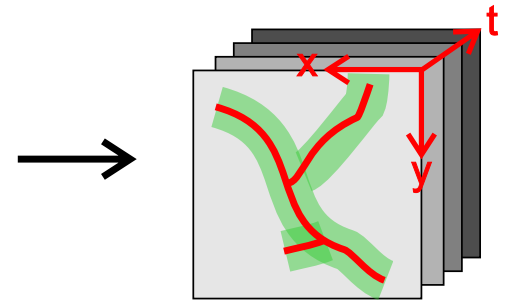

Time series of endfoot $\mathrm{Ca}^{2+}$ events

B

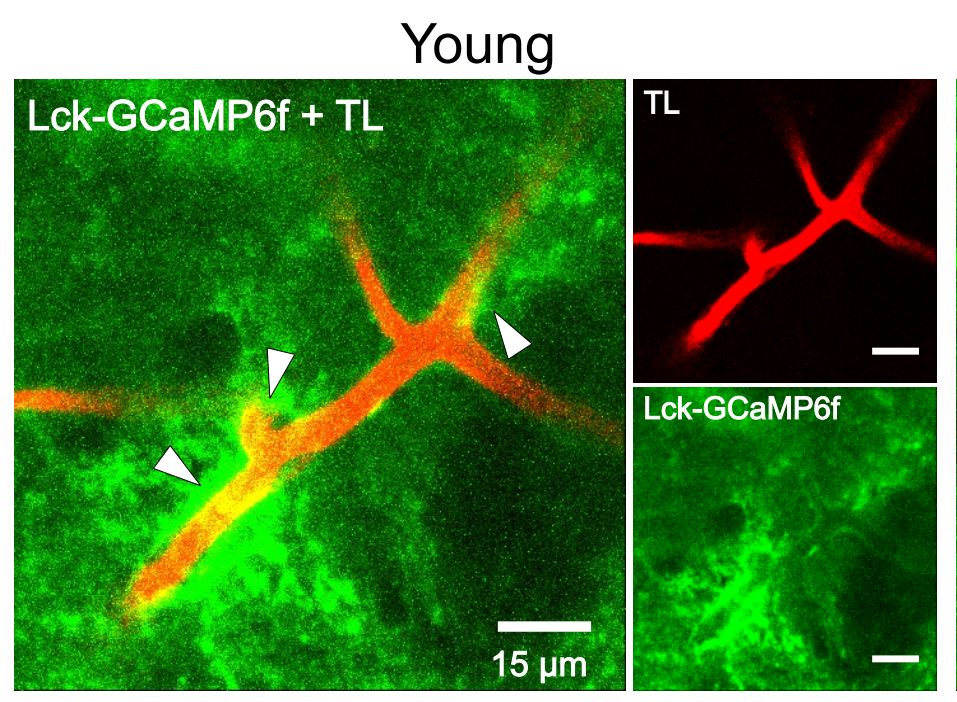

Aging
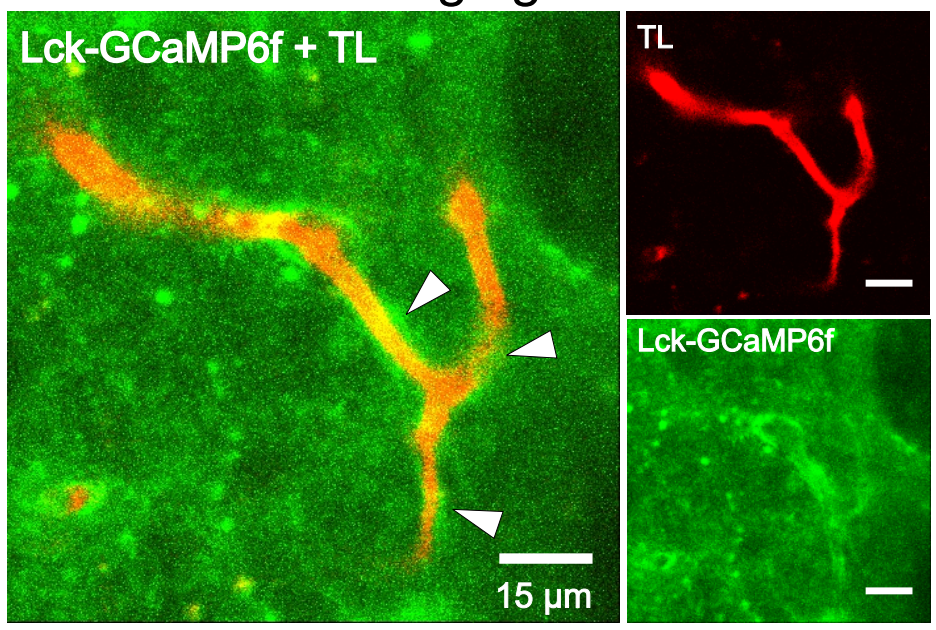

C

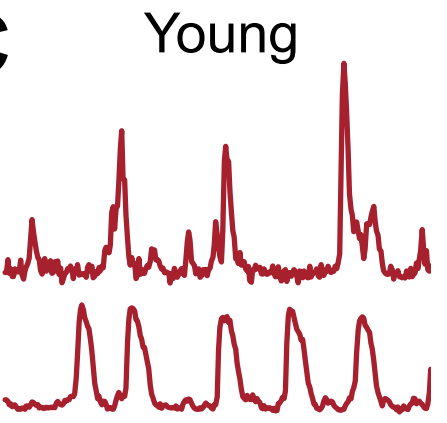

Aging

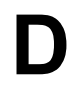

n.s. $p=0.124$

** $p=0.002$

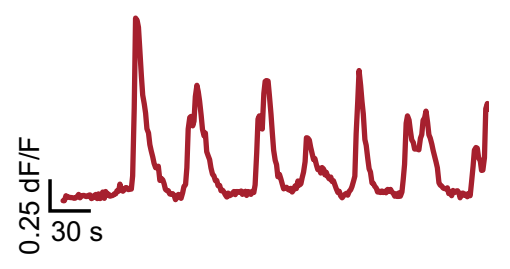

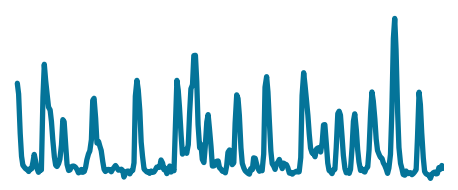

combinde
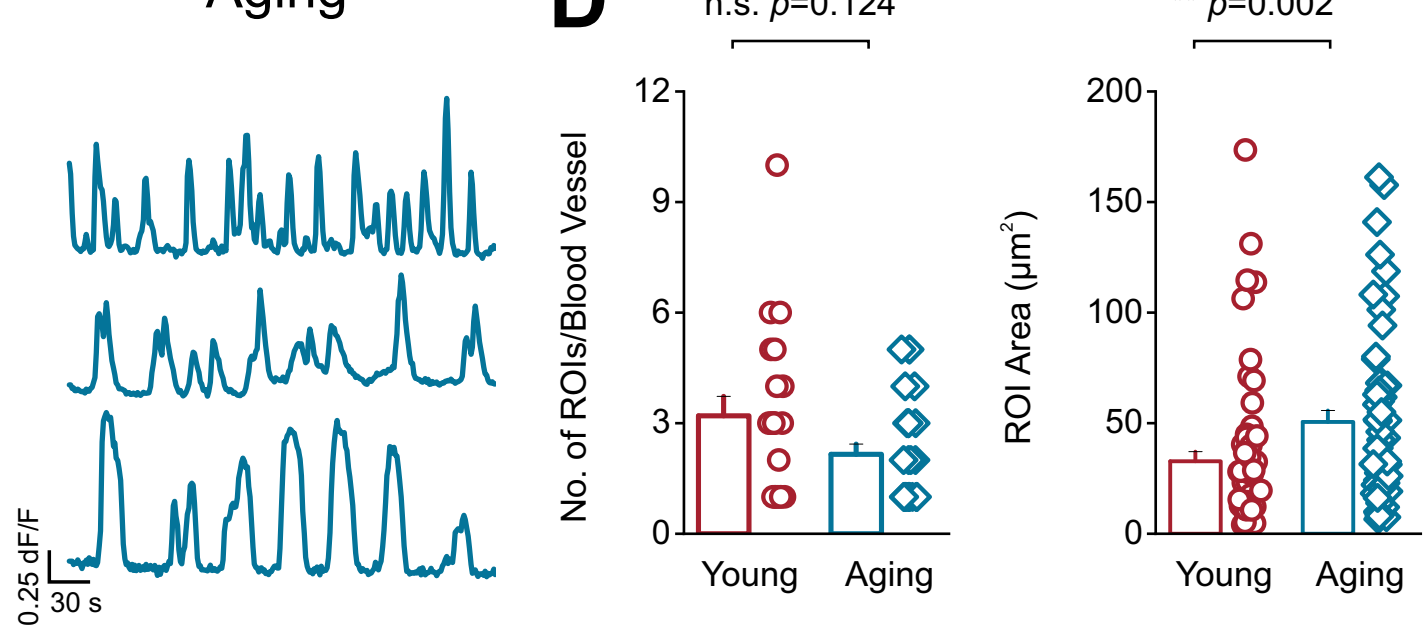
Figure 2 available under aCC-BY-NC-ND 4.0 International license.
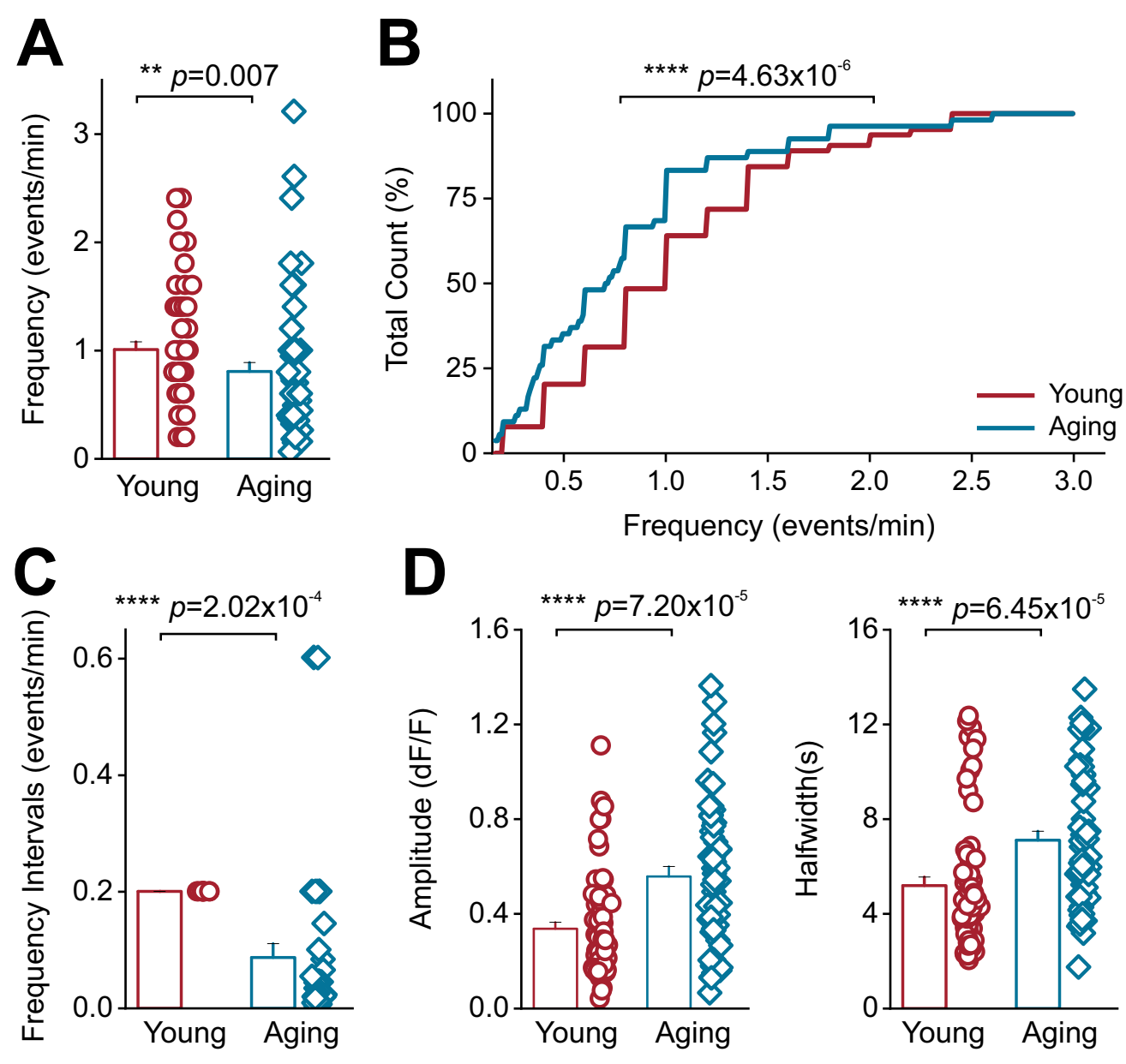
Figure 3 available under aCC-BY-NC-ND 4.0 International license.
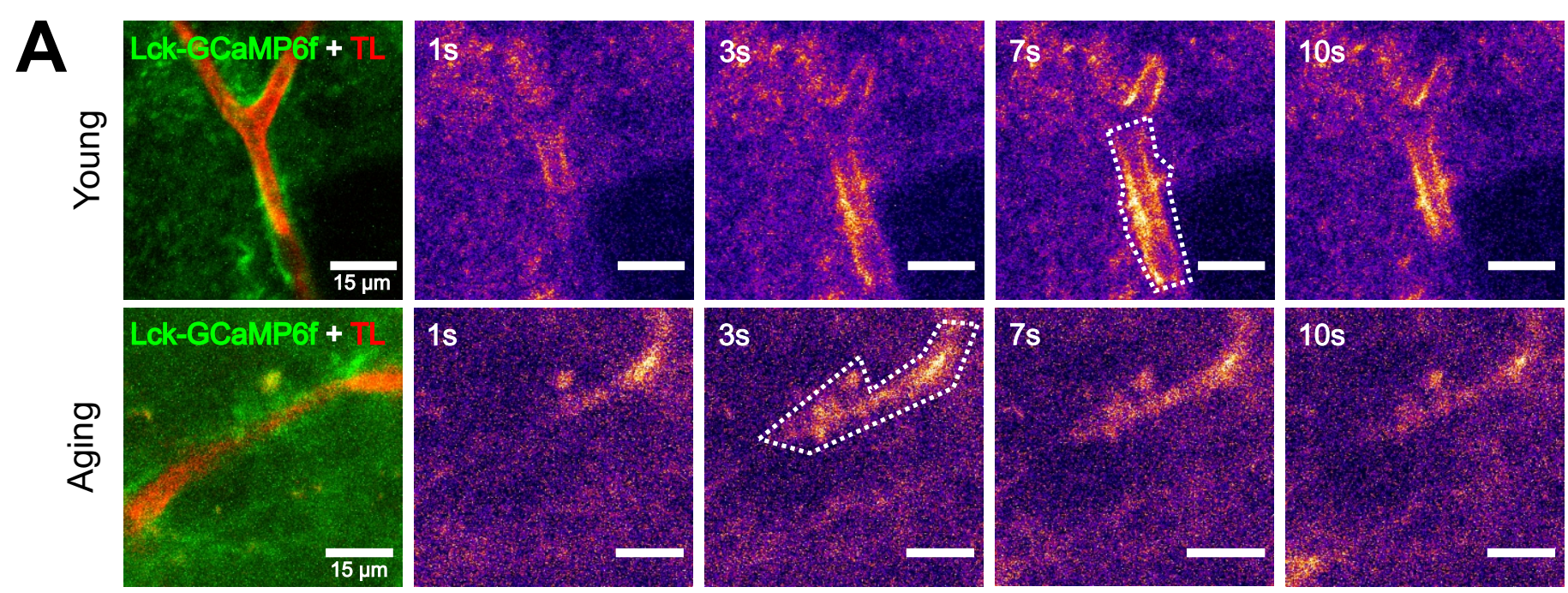
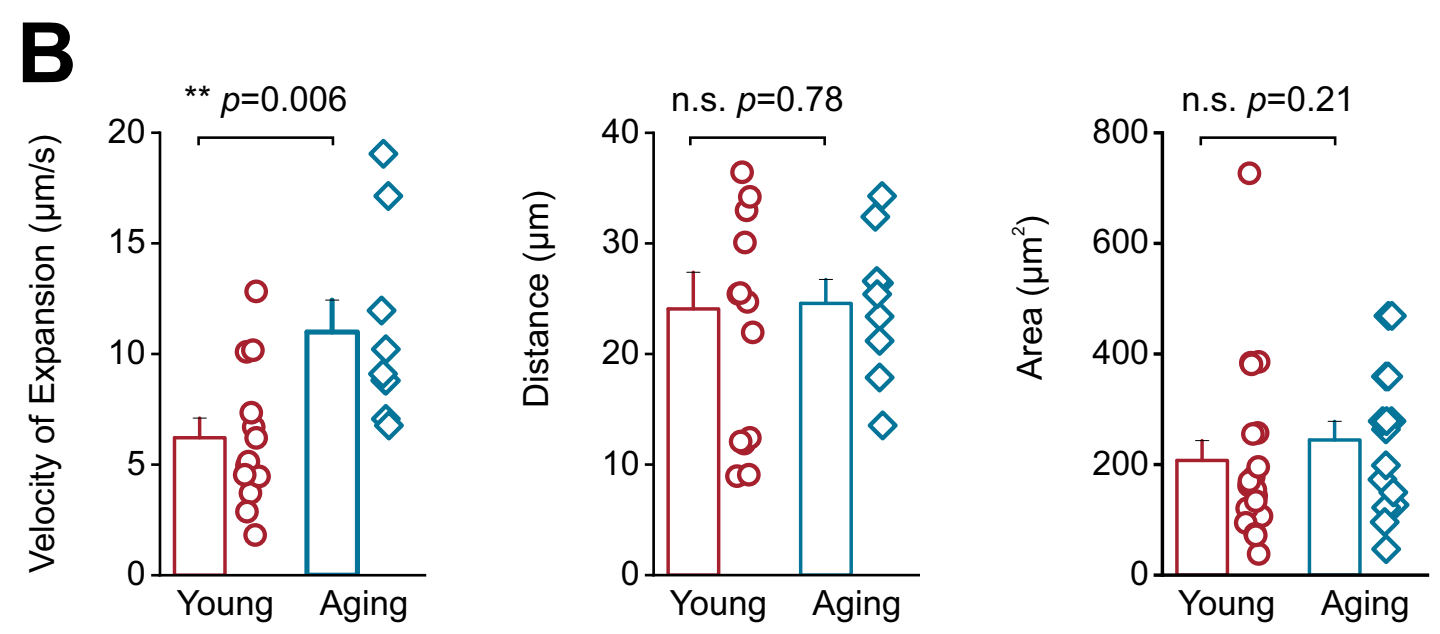
$\mathbf{A}$

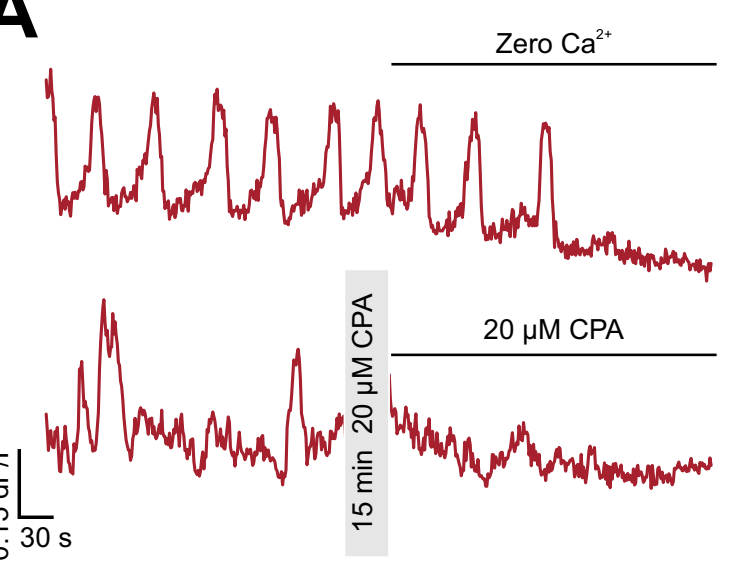

D
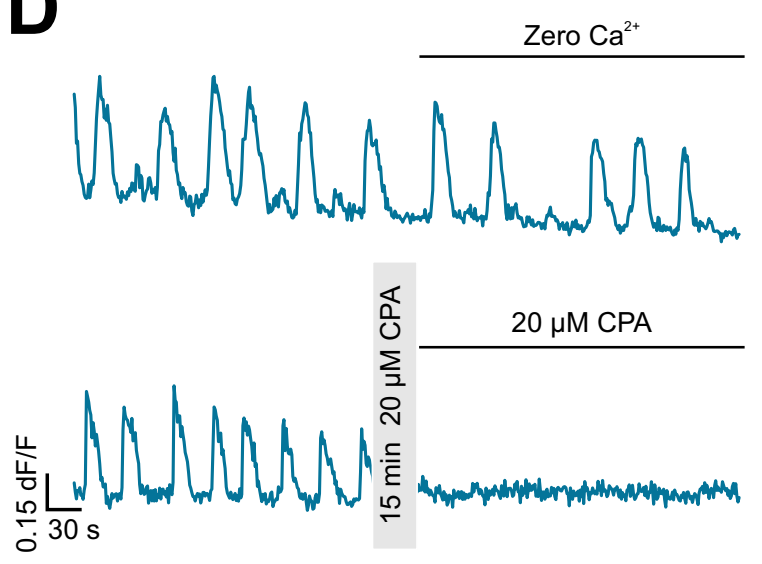

B

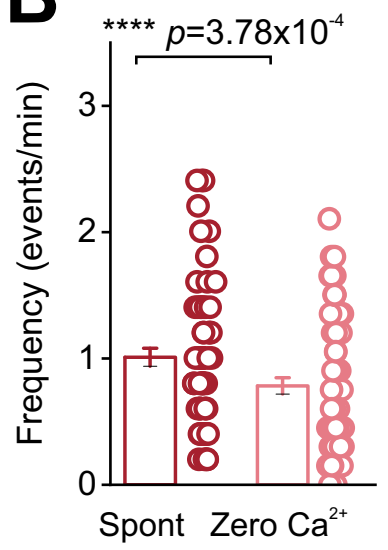

Aging
E

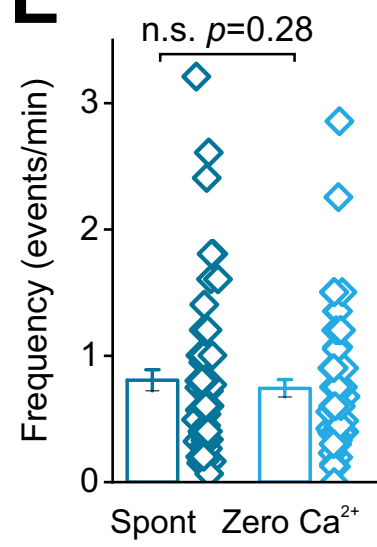

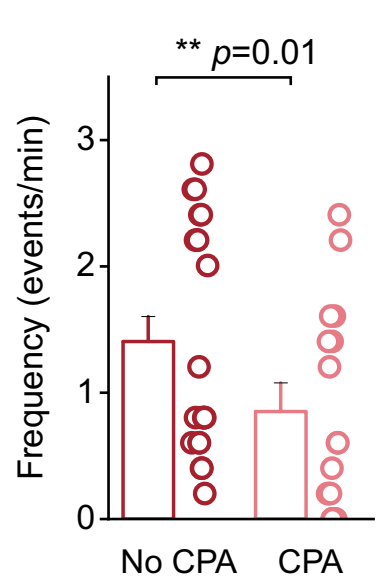

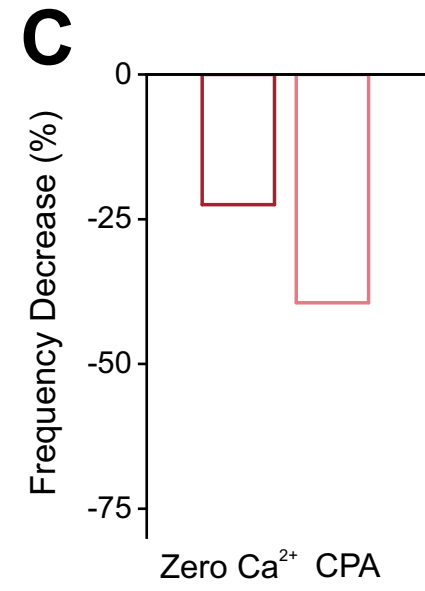

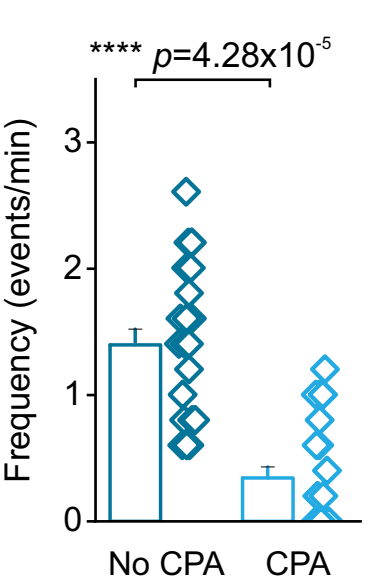

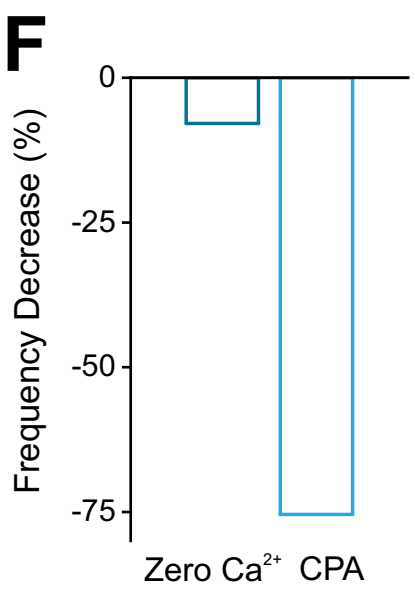




\section{Young}

A
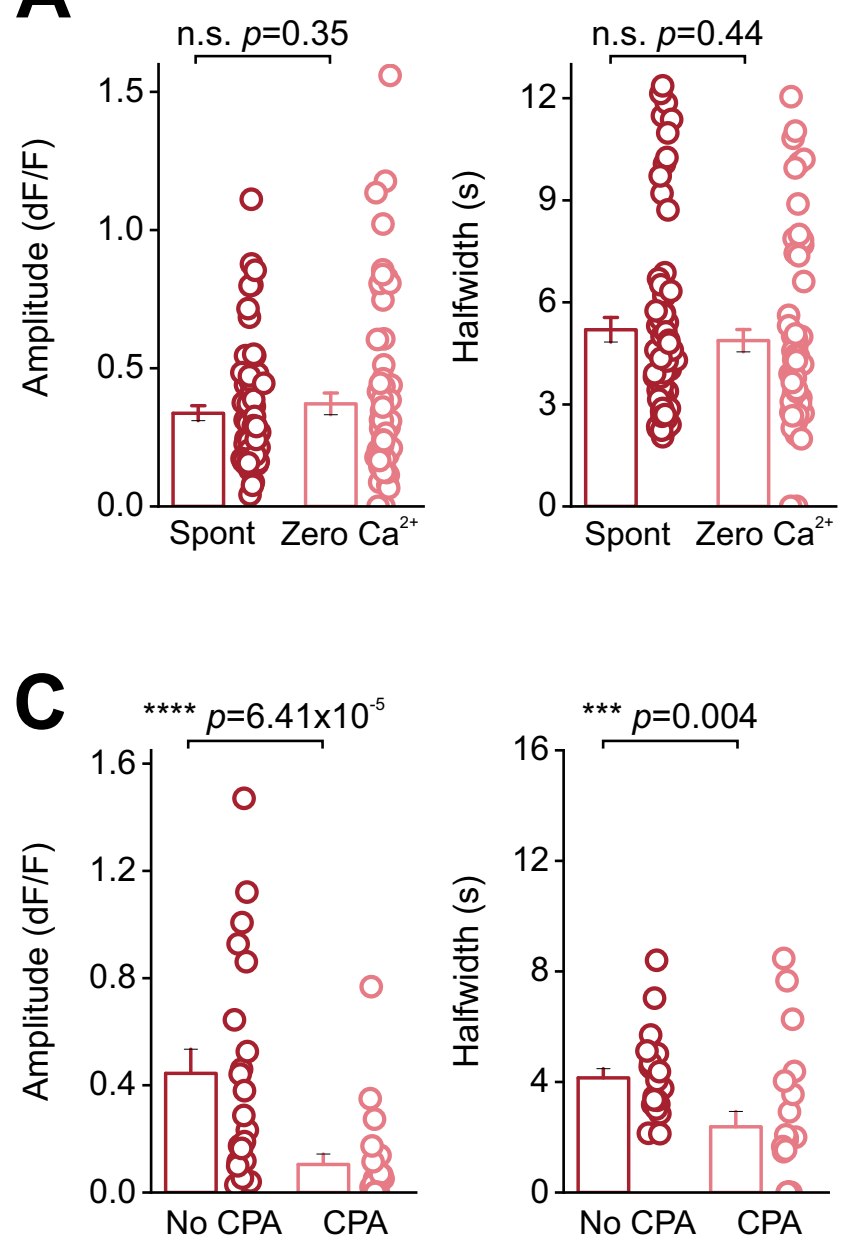

Aging
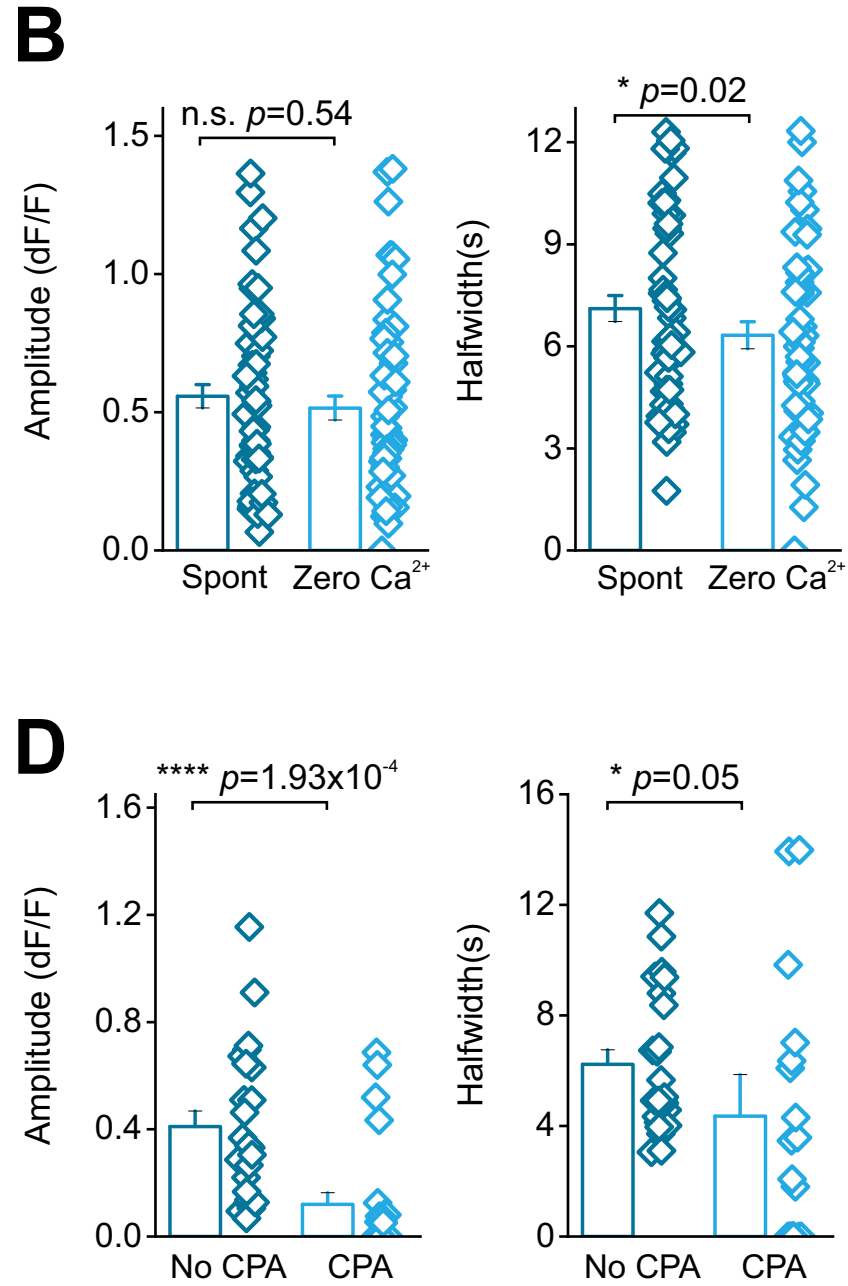
Figure 6 available under aCC-BY-NC-ND 4.0 International license.
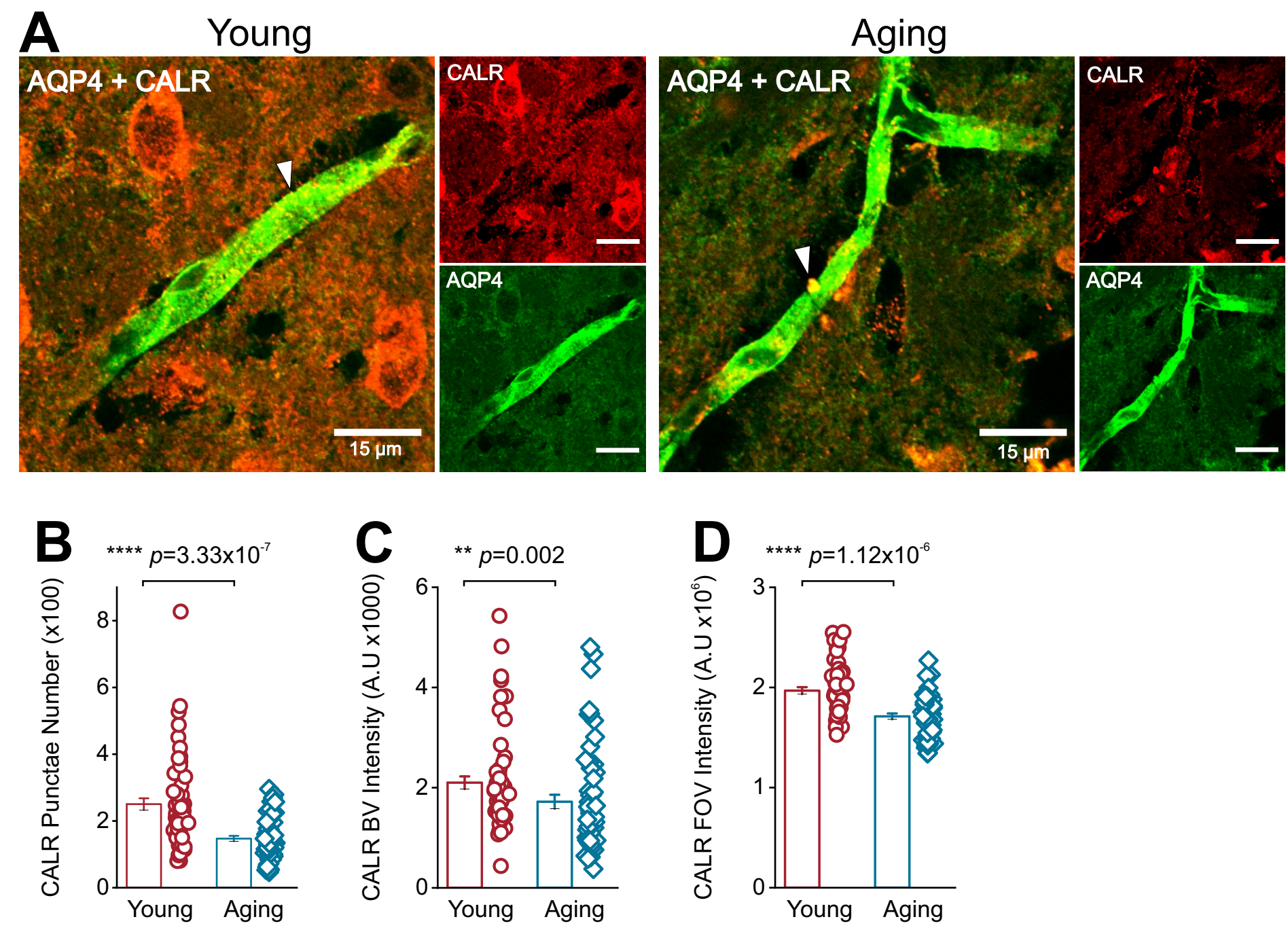
Figure 7 available under aCC-BY-NC-ND 4.0 International license.

A Injection of AAV into DLS

Confocal Imaging

GfaABC1D-mito7-GCaMP6f

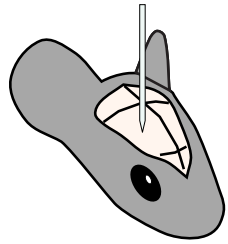

Young:

3-4 months old 20-24 months old
3 weeks

Intraventricular injection of TL

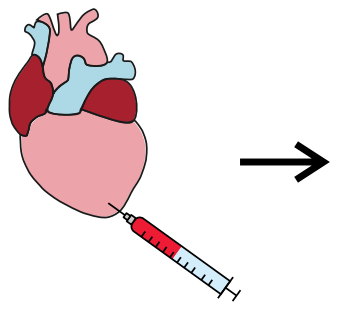

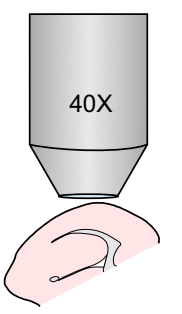

Imaging of striatal brain slice
Analysis

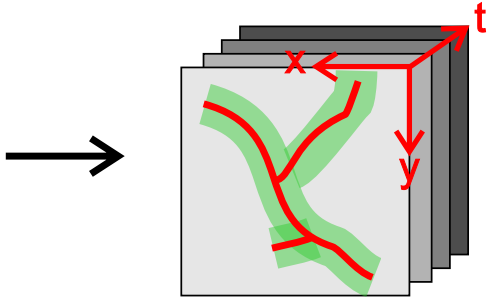

Time series of endfoot $\mathrm{Ca}^{2+}$ events
B

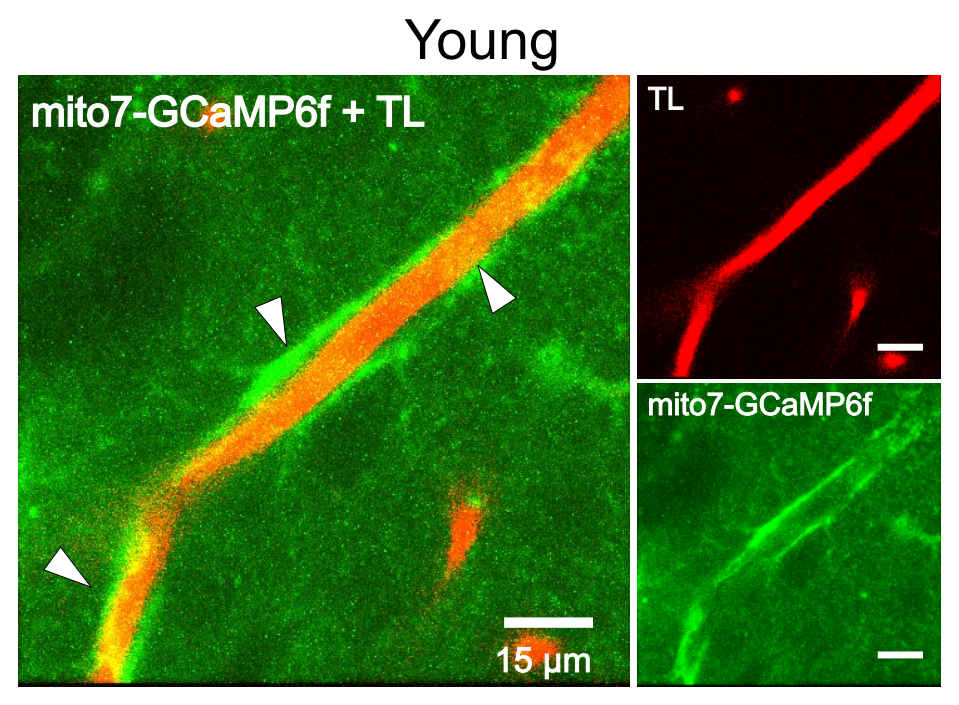

C

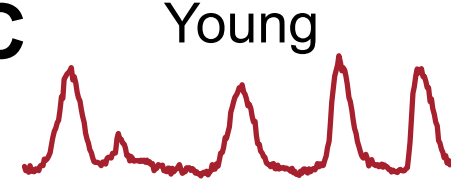

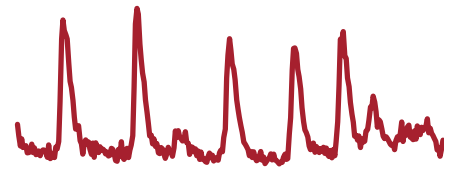

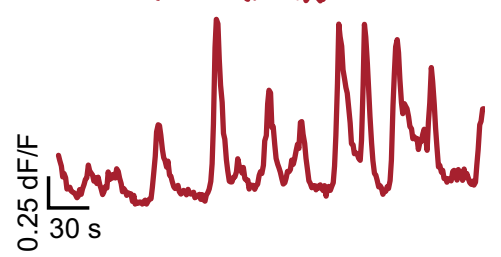

Aging

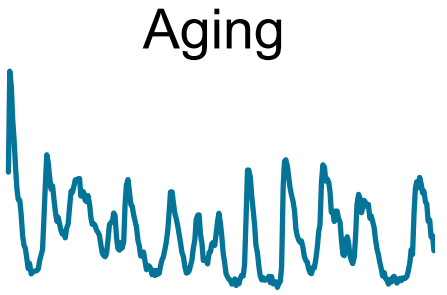

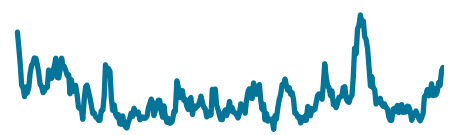

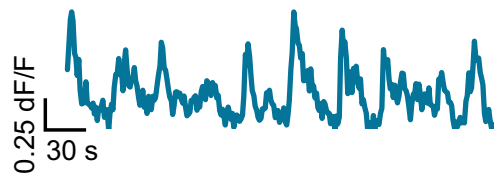

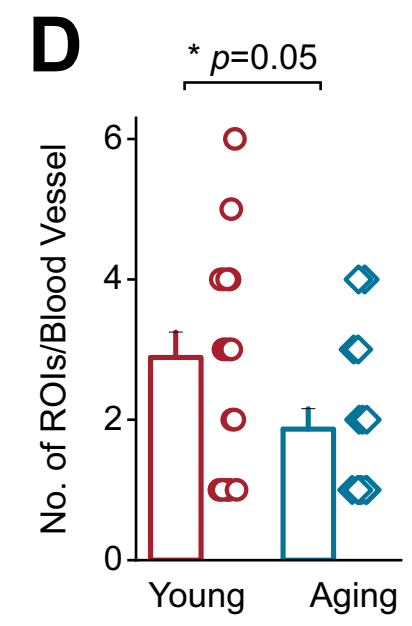

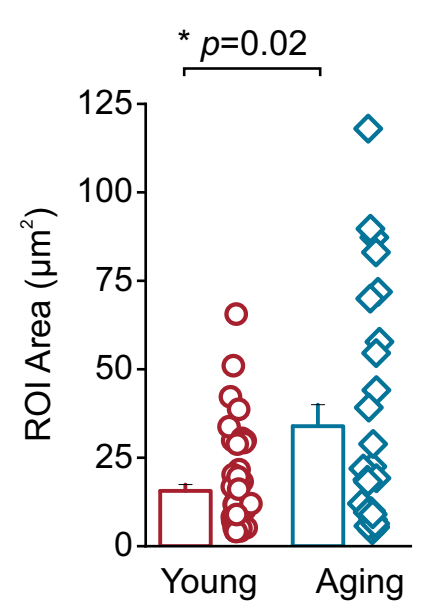

Aging
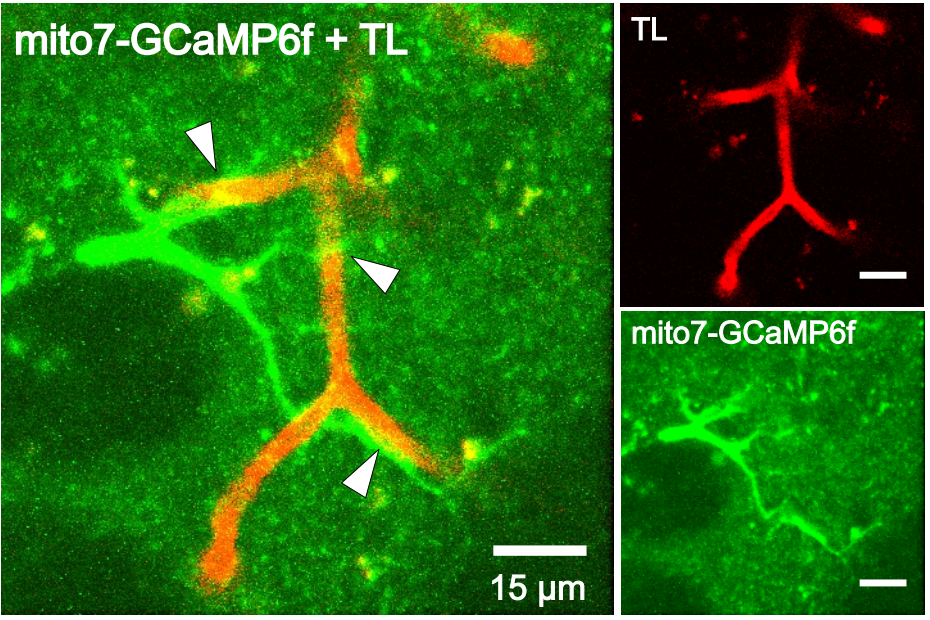

Figure 8
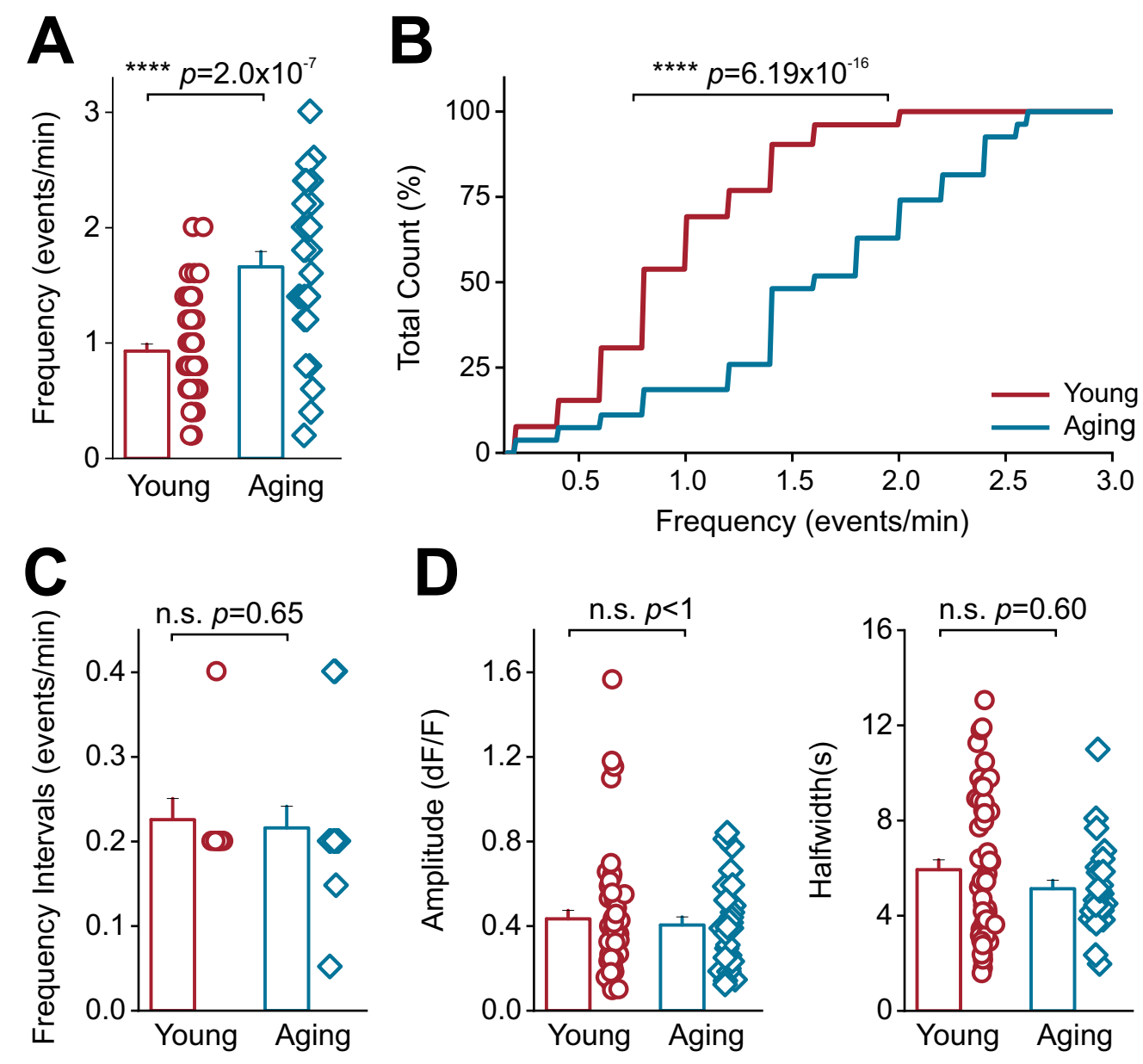
A

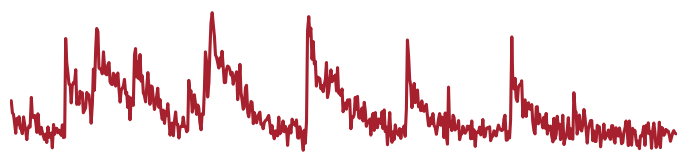<smiles></smiles><smiles>[Na][Mg][Mg][Mg]</smiles>

C

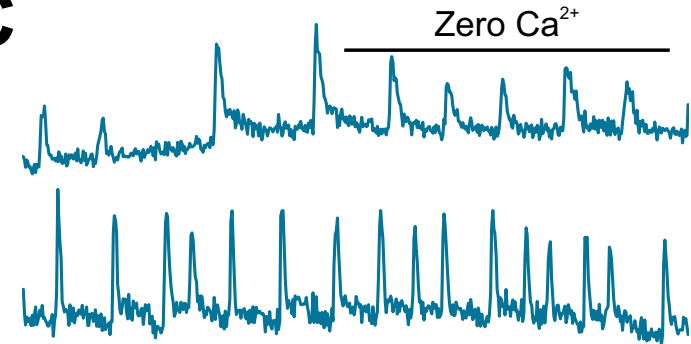

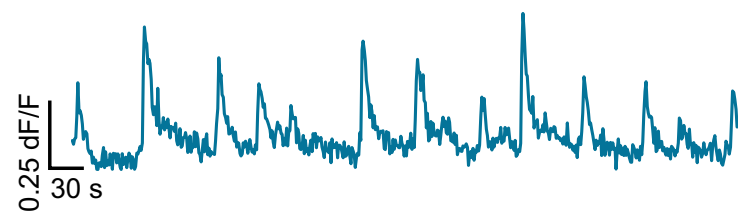

\section{Young}
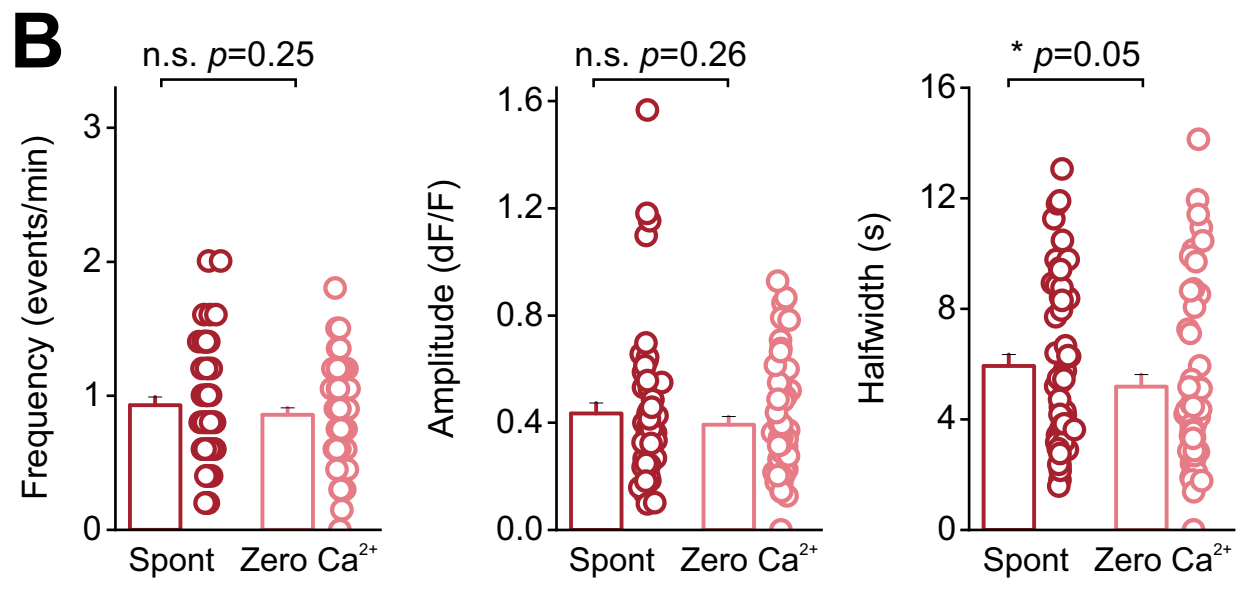

Aging
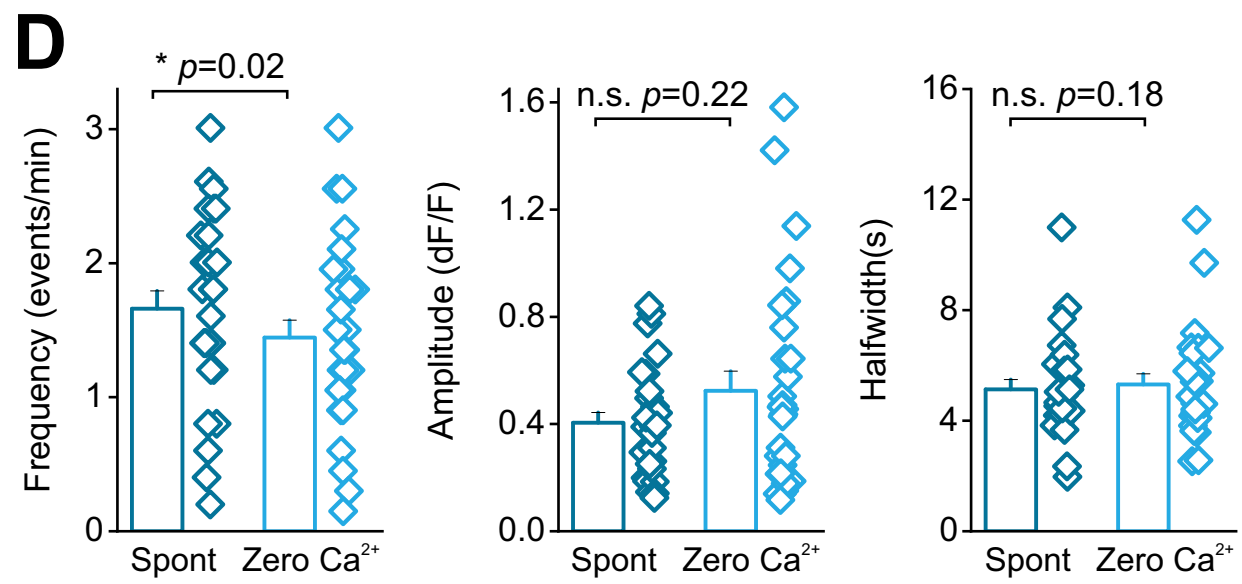
bioRxiv preprint doi: https://doi.org/10.1101/2021.09.24.461710; this version posted September 25, 2021. The copyright holder for this preprint (which was not certified by peer review) is the author/funder, who has granted bioRxiv a license to display the preprint in perpetuity. It is made Figure 10

Young
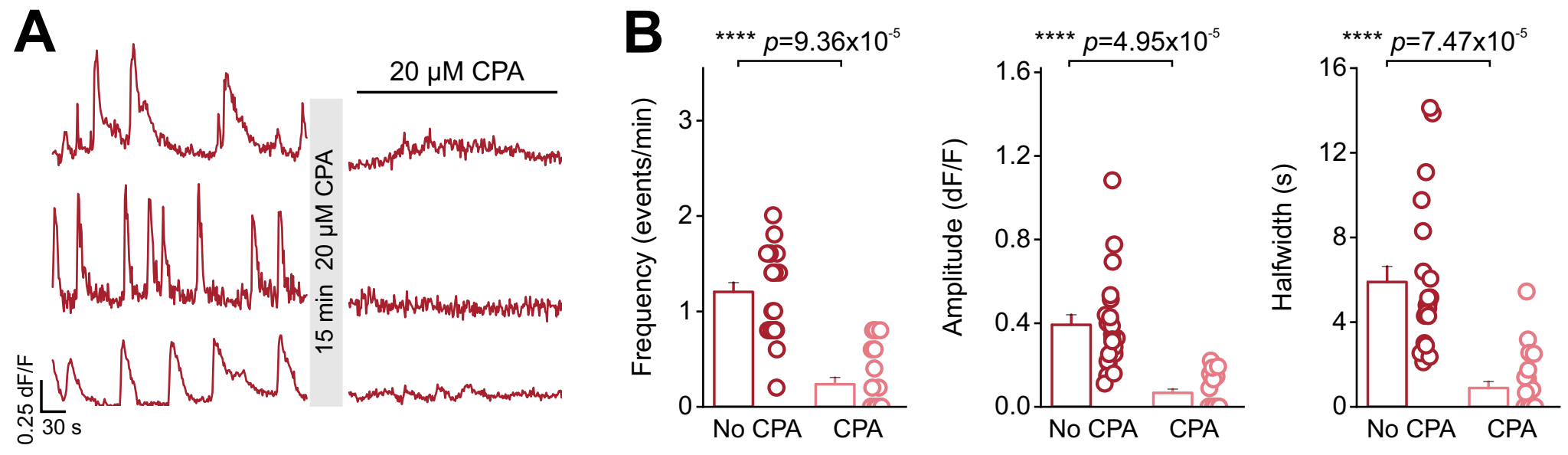

C
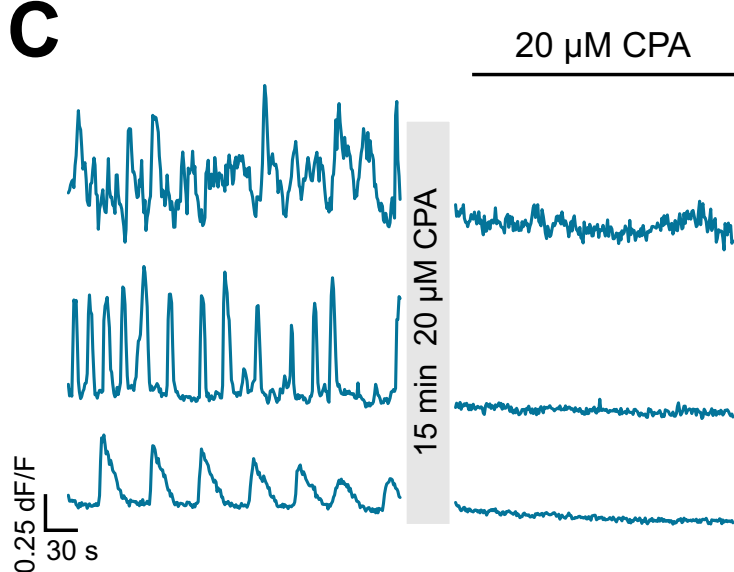

Aging

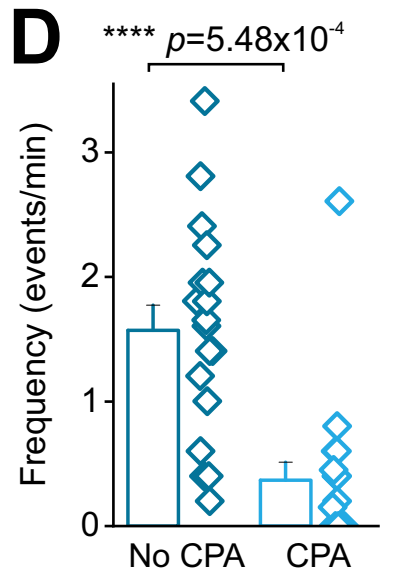

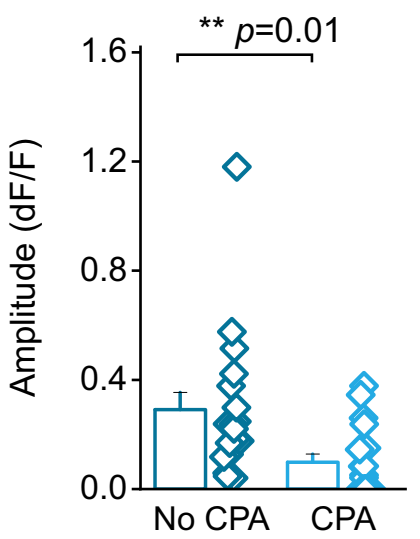

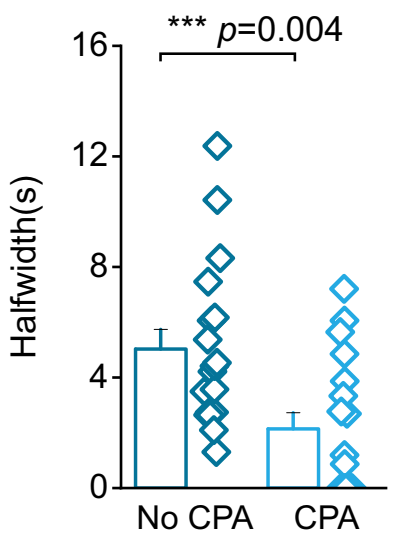

